\title{
FINITELY GENERATED KLEINIAN GROUPS IN 3-SPACE AND 3-MANIFOLDS OF INFINITE HOMOTOPY TYPE
}

\author{
L. POTYAGAILO
}

\begin{abstract}
We prove the existence of a finitely generated Kleinian group $N \subset$ $S O_{+}(1,4)$ acting freely on an invariant component $\Omega \subset S^{3}$ without parabolic elements such that the fundamental group $\pi_{1}(\Omega / N)$ is not finitely generated.

Moreover, $N$ is a finite index subgroup of a Kleinian group $N_{0}$ which has infinitely many conjugacy classes of elliptic elements.
\end{abstract}

\section{INTRODUCTION}

1.1. Formulation of results. We will consider finitely generated Kleinian (discontinuous) subgroups of the conformal group $M(n) \cong S O_{+}(1, n+1)$ acting on the $n$-dimensional sphere $S^{n}=R^{n} \cup\{\infty\}$. As usual, let $\Omega(G) \subset S^{n}$ denote the domain of discontinuity and $\Lambda(G)=S^{n} \backslash \Omega(G)$ denote the limit set of the group $G \subset M(n)$.

A Kleinian group $G \subset M(n)$ is called a function group if there exists a connected component $\Omega_{G} \subset \Omega(G)$ invariant under $G$. See [M2] for standard material on Kleinian group theory. In the present paper we prove the following:

Theorem A. There exists a finitely generated function group $N \subset M(3)$, without parabolics, acting freely on an invariant component $\Omega_{N} \subset S^{3}$ such that the group $\pi_{1}\left(\Omega_{N} / N\right)$ is not finitely generated.

To be hyperbolic in the sense of $\mathrm{M}$. Gromov [G] is an important property of finitely generated groups. In particular, it follows from [Sc1] and [T2] that a finitely generated Kleinian group acting on $\mathbf{C}$ and containing no parabolic elements is Gromov hyperbolic. Let us notice that if $G \subset M(n)$ contains a parabolic subgroup of the rank greater than 1 then $G$ is not Gromov hyperbolic [G, p. 108].

The following result shows that the situation in higher dimensions is quite different.

Theorem B. The group $N$ is a finite index subgroup of a group $N_{0}$ which contains infinitely many conjugacy classes of elements of finite order. have

Due to well-known properties of Gromov hyperbolic groups [G, p. 102] we

Received by the editors June 4, 1991.

1991 Mathematics Subject Classification. Primary 57M10, 30F40, 20H10; Secondary 57S30, 57M05, 30F10, 30F35.

Key words and phrases. Kleinian groups, conformal group of euclidean space, uniformization of 3-manifolds, surface bundle over the circle. 
Corollary 1. The groups $N_{0}$ and $N$ are not Gromov hyperbolic.

The following corollary immediately follows from the proof of Theorem B (see $\S 2)$.

Corollary 2. There exist a Gromov hyperbolic group $G^{*} \subset M(3)$, without torsion, containing $N$ as a normal subgroup.

1.2. Historical remarks. In [A], L. Ahlfors proved his famous finiteness theorem: that is a finitely generated nonelementary Kleinian group $G \subset M(2)$ has a factor-space $\Omega(G) / G$ consisting of a finite number of Riemann surfaces $S_{1}, \ldots, S_{n}$ each having a finite hyperbolic area.

There are now topological approaches to finiteness statements for Kleinian groups acting on hyperbolic 3-space and its boundary. Let us point out some of them; P. Scott [Sc1] showed that each 3-manifold with a finitely generated fundamental group $\pi_{1}$, contains an embedded compact 3-manifold ("core") such that the embedding induces an isomorphism on the fundamental groups (as a corollary $\pi_{1}$ is finitely presented). By the well-known Selberg lemma [Se], each finitely generated Kleinian group $G \subset M(2)$ contains a torsion-free subgroup of finite index and hence, by Scott's "core" theorem, $G$ is also finitely presented.

By using the "core" theorem, R. Kulkarni and P. Shalen obtained a weak version of Ahlfors' finiteness theorem [Ku-S]. D. McCullough and M. Feighn improved the last result in $[\mathrm{Mc}-\mathrm{F}]$ by using a relative version of the "core" theorem, due to D. McCullough [Mc]. M. Feighn and G. Mess [F-M] showed that the number of conjugacy classes of finite subgroups of a finitely generated discontinuous group contained in $\mathrm{PSL}_{2} \mathrm{C}$ is finite.

It was proven by $\mathrm{F}$. Bonahon [B] that the interior of a hyperbolic 3-manifold with indecomposable finitely generated fundamental group is homeomorphic to the interior of Scott's core.

In higher dimensions the situation is changed dramatically. We showed in [K-P] that even the weakest version of Ahlfors' finiteness theorem does not hold for Kleinian groups acting on $S^{3}$. Moreover, there exists a finitely generated Kleinian group which admits no finite presentation and, so, there is a hyperbolic 4-manifold without compact "core" [P].

M. Kapovich constructed [K] a finitely generated Kleinian group $\Gamma \subset M(3)$ which contains infinitely many $\Gamma$-conjugacy classes of maximal parabolic subgroups and elliptic elements. This result shows that Sullivan's finiteness theorem for cusps [Su2] (see also [Mc-F]) fails in higher dimensions.

The common point of all of these counterexamples to a version of the finiteness theorem in higher dimensions was the availability of parabolic elements. Moreover these constructions used accidental parabolics which do not correspond to cusp ends of the quotient of an invariant component [K-P].

An important problem arising here is to describe the class of finitely generated Kleinian groups in higher dimensions having a factor space of finite homotopy type. It was conjectured that all counterexamples to a version of the finiteness theorem in higher dimensions are connected either with the existence of parabolic elements or with algebraic structure of a Kleinian group (see discussion in $[\mathrm{K}]$ ).

The construction of the group $N$ given in Theorem A will be obtained in $\S 3$ 
and it uses new ideas compared with [K-P]. We give a sketch of the proof of Theorems $\mathrm{A}$ and $\mathrm{B}$ in $\S 2$.

Theorems $\mathrm{A}$ and $\mathrm{B}$ have been announced in $[\mathrm{P}]$.

As I learned after I finished this work, B. Bowditch and G. Mess constructed by a different method an interesting example of a finitely generated but infinitely presented Kleinian group in $M(3)$ without parabolic elements. Our Theorem $A$ and the main result in [Bow-M] intersect but do not overlap.

\section{ACKNOWLEDGMENTS}

I would like to thank Michael Gromov and Michael Kapovich for useful discussions. The author is deeply grateful to the referee for several useful corrections improving the paper.

\section{OUtLine OF THE PROOFS OF TheOREMS A AND B}

We shall consider the family of Euclidean spheres $\Sigma_{1}, \ldots, \Sigma_{s}, \ldots, \Sigma_{2 s}$ covering the square knot, $K$, which is a connected sum of trefoils along a sphere $\sigma$ (see Figure 1). Each $\Sigma_{i}$ is a result of a reflection $\tau_{i-1}$ in a plane $\Pi_{i-1}$ of the previous sphere $\Sigma_{i-1}$ and $\Sigma_{2 s+1-i}=\tau_{\sigma}\left(\Sigma_{i}\right)$ where $\tau_{\sigma}$ is the reflection in $\sigma \quad(i=1, \ldots, s)$. We suppose that a group $\Gamma_{1} \subset \operatorname{PSL}_{2}(\mathbf{C})$ acts in the exterior of the sphere $\Sigma_{1}$ so that $\Lambda\left(\Gamma_{1}\right)=\Sigma_{1}$. The group $\Gamma_{1}$ will be commensurable to the reflection group determined by the faces of a right angled dodecahedron. The choice of $\Gamma_{1}$ is described more concretely in the next paragraph and is to be conditioned by the following:

(i) There is a normal finitely generated subgroup $F_{1} \triangleleft \Gamma_{1}$ of infinite index.

(ii) There exists a subgroup $\Gamma_{i}^{\prime} \subset \Gamma_{i}$ isomorphic to the fundamental group of a compact hyperbolic 3-manifold with geodesic boundary such that the group $G=\left\langle\Gamma_{1}^{\prime}, \Gamma_{2}^{\prime}, \ldots, \Gamma_{2 s}^{\prime}\right\rangle$ is discontinuous. Here $\Gamma_{i}=\tau_{i-1} \Gamma_{i-1} \tau_{i-1} \quad(i=2, \ldots$, $2 s)$.

(iii) There is a normal, finitely generated, nontrivial subgroup $N \triangleleft G^{*}$ of infinite index in a group $G^{*} \subset G$ such that the index $\left|G: G^{*}\right|<\infty$.

We will construct an incompressible torus $T \subset R(G) \subset \Omega_{G}$ where $R(G)$ is a fundamental domain of the group $G$ in a component $\Omega_{G}\left(\infty \in \Omega_{G}\right)$. Due to the equality $\Omega(N)=\Omega(G)=\Omega\left(G^{*}\right)$ we have a diagram of coverings

$$
\Omega_{G} \stackrel{p_{1}}{\longrightarrow} M_{N} \stackrel{p_{2}}{\longrightarrow} M_{G^{*}}
$$

We will prove that $G$ is geometrically finite and contains no parabolics and, hence, that the manifold $M_{G^{*}}$ is compact.

Both coverings $p_{i}$ are infinite; by the construction the group $N$ is normal in $G^{*}$, it now easily follows that the group $\pi_{1}\left(M_{N}\right)$ is a normal subgroup of infinite index in $\pi_{1}\left(M_{G}^{*}\right)$, due to the fact that $p_{2 *}\left(\pi_{1} M_{N}\right)$ contains the kernel of the natural epimorphism $\pi_{1}\left(M_{G^{*}}\right) \rightarrow G^{*}$. By [He, Theorem 11.1], either the group $\pi_{1}\left(M_{N}\right)$ is infinitely generated or is isomorphic to a surface group. But the second is impossible, since $\pi_{1}\left(M_{N}\right)$ is not virtually $\mathbf{Z} \oplus \mathbf{Z}$.

Informal description of the construction. ${ }^{1}$ We will construct a 1-parameter family of convex cocompact groups $G(t)$ for $t \in[-\varepsilon, 0]$. There is a limiting group $G(0)$ which is geometrically finite but has a parabolic element, and the family

\footnotetext{
${ }^{1}$ This is an informal description of our construction; the reader who prefers exact explanation may skip this paragraph.
} 
can be extended to give groups $G(t)$ for $t>0$; for $t=\pi / 2 n$ ( $n$ is sufficiently large) these groups are discrete. Although the actual construction produces just one of these groups, it will help in following the construction to think of this group as the end of a process.

We start with quasifuchsian group $G_{s}$ having the limit set $\Lambda\left(G_{s}\right)$ which is a 2-dimensional quasisphere embedded in $S^{3}$. The group $G_{s}$ contains a fuchsian subgroup $\mathscr{H}_{s}$ leaving invariant the plane $\Pi_{s}$ and all spheres containing the circle $\Lambda\left(\mathscr{H}_{s}\right)$-its limit set. We first consider the action of $G_{s}$ on its interior invariant component $\Omega_{s}^{\prime}$ not containing $\infty$. There is a family of spheres $P(\theta)$ $\left(\theta_{0} \leq \theta \leq \pi / 2\right)$ between $\Pi_{s}=P\left(\theta_{0}\right)$ and $P(\pi / 2)$ such that $P(\theta)$ makes an angle $\theta$ with $\partial B_{s}$, and all $P(\theta)$ contains $\Lambda(\mathscr{H}(G))$.

We choose $\theta$ to be close to $\pi / 2$ and set $\Omega_{s}^{-}=\Omega \backslash G \cdot(\operatorname{int}(P(\theta)))$, which is a manifold with boundary $\partial \Omega_{s}^{-}, \Omega_{s}^{-} \backslash \partial \Omega_{s}^{-}$is an open ball. A subgroup $G_{s}^{\prime} \subset G_{s}$ which keeps $\Omega_{s}^{-}$invariant has a fundamental domain $R_{1} \subset \Omega_{s}^{-}$. Denote by $R_{2}$ the double of $R_{1}$ across $P(\theta)$. It is the fundamental domain for a group $G(-\varepsilon)$, which is a double $G_{s}^{\prime} * \mathscr{H}_{s} G_{s}^{\prime}$ of the group $G_{s}^{\prime}$ along the subgroup $\mathscr{H}_{s}$ (the other copy of $G_{s}^{\prime}$ is the conjugate of $G_{s}^{\prime}$ by reflection in the plane $P(\theta)$ ).

Roughly speaking, both $R_{2}$ and the closed quasiball $\mathrm{cl} G(-\varepsilon) \cdot R_{2}$ look like a horseshoe with a trefoil tied in each arm, and bent so the arms are quite close. The construction ensures that $\partial R_{2}$ will be near to the limit set $\Lambda(G(-\varepsilon))$, which is quasisphere $\partial\left[\mathrm{cl} G(-\varepsilon) \cdot R_{2}\right]$.

There is also a fundamental domain $R_{3}$ for the action of $G(-\varepsilon)$ on the exterior of $\Lambda(G(-\varepsilon))$. The boundary of $R_{3}$ consists of isometric spheres, $R_{3}$ looks like a ball into which two wormholes have been dug. Each wormhole is knotted, but has a dead end. The tunnels fork slightly before their ends, so on $\partial R_{3}$ there are two pairs of faces: $\left(F_{g_{1}}, F_{g_{2}}\right)$ and $\left(F_{g_{1}}^{-1}, F_{g_{2}}^{-1}\right)$ where $F_{g_{1}}=I_{g_{1}(-\varepsilon)} \cap \partial R_{3}$ and $F_{g_{2}}=I_{g_{2}(-\varepsilon)} \cap \partial R_{3}$, and similarly for $F_{g_{1}}^{-1}$ and $F_{g_{2}}^{-1}$.

Given two spheres $P, Q$ in $S^{3}$, we say that the conformal distance between $P$ and $Q$ is $\varepsilon$ if $\varepsilon$ is the distance between the totally geodesic hyperplanes which the spheres bound in $\mathscr{H}^{4}$ (if these are disjoint) or the negative of the angle between $P$ and $Q$ if the spheres meet.

Now let $R_{2}(t(\theta))$ be the double of $R_{1}$ across the sphere $P(t(\theta))$ for a function $t=t(\theta)$ to be defined. The domain $R_{2}(t(\theta))$ is fundamental inside a quasisphere $\Lambda(G(t))$, and there is a fundamental domain $R_{3}(t)$ for $G(t)$ in the exterior of $\Lambda(G(t))$, which has two faces $F_{g_{1}}(t), F_{g_{2}}(t)$ and $F_{g_{1}^{-1}}(t), F_{g_{2}^{-1}}(t)$ so that each pair has conformal distance $-t$. When $t$ becomes zero the spheres $F_{g_{1}}(t)$ and $F_{g_{2}}(t)$ touch at a point $p$ and the spheres through the faces $F_{g_{1}^{-1}}, F_{g_{2}^{-1}}$ touch at a point $q$. The points $p$ and $q$ belong to the limit set not to $R_{3}(0)$. When the faces of $R_{3}(0)$ are glued up, we obtain a noncompact 3-manifold. Note that $R_{2}(0)$ is a compact ball, and for sufficiently small $t, R_{3}(t)$ remains a compact ball. In $\operatorname{cl}\left(R_{3}(0)\right), p$ has a neighbourhood $N(p)$ which is a solid of revolution: $N(p) \backslash\{p\}=S^{1} \times V$ where $V \subset \mathbf{C}$ is the strip $y \geq 1,0 \leq x \leq c$ for some $c$. The domain $N(p) \backslash\{p\} \cup N(q) \backslash\{q\}$ projects to a cusp region $S^{1} \times S^{1} \times[0, \infty)$ conformally equivalent to a cusp end of a hyperbolic manifold. We can consider the manifolds $M(t)$ for $t<0$ as the result of conformal Dehn surgery on the manifold $M(0)$; this conformal Dehn surgery generalizes Thurston's hyperbolic Dehn surgery. If we continue to deform the group, the pairs of faces meet at the angle $t$ instead of having conformal distance $-t>0$. 
When $t=2 \pi / n$ and $n$ is large enough, the group $G(t)$ is discrete. In the fundamental domain $R_{3}(t)$, the two knotted wormholes have met, so $R_{3}(t)$ is almost a "cube with a knotted hole"; in fact the boundary has genus 2, but if it is compressed we get a square knot exterior. When we have shown that the torus is incompressible, Theorem A will follow at the same time we will have constructed the example for Theorem B.

\section{Preliminary Results}

Let us consider a piecewise linear curve $K_{1}$ representing the trefoil (Figure 1a). Let $B_{1}$ be a Euclidean ball centered on $K_{1}$ and planes $\Pi_{i}$ are orthogonal to $K_{1}$. Denote $D_{i}=\Pi_{i} \cap B_{1} \quad(i=1,2)$.

We say that two disjoint Euclidean disks $D_{i}$ are opposite if $D_{i} \subset B_{1}$ and the diameter $d$ of $B_{1}$ which is the common perpendicular to $D_{i}$ has $d \cap D_{i}$ as (Euclidean) center of $D_{i}(i=1,2)$. The following is purely geometrical.

Lemma 1. Let us suppose that there exist three pairs $\left(D_{j}, D_{j}^{\prime}\right)$ of mutually disjoint opposite disks in $B_{1}$ such that the common perpendiculars $d_{j}$ for each pair do not lie in a single 2-plane $(j=1,2,3)$. Then there exists a covering

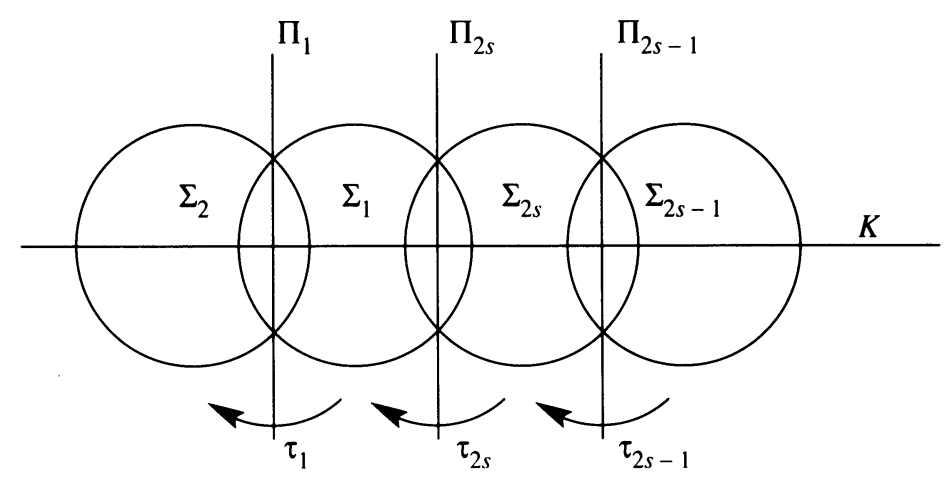

Figure 1a

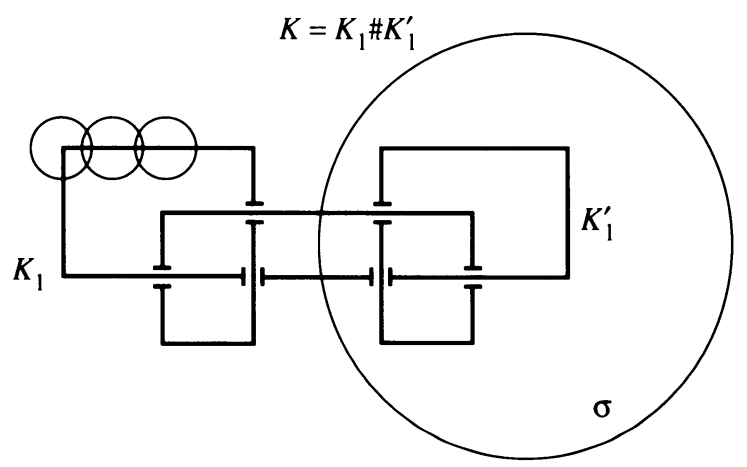

Figure 1b 
$B=\left\{B_{1}, \ldots, B_{n}\right\}$ of $K_{1}$ by balls, where each $B_{i}$ is image under reflection $\tau_{i-1}$ in the plane $\Pi_{i-1}$ of $B_{i-1}$. Here $\Pi_{1}$ and $\Pi_{0}$ are planes containing $D_{j}$ or $D_{j}^{\prime}$ and $\Pi_{i}=\tau_{i-1}\left(\Pi_{i-2}\right), \Pi_{n}=\Pi_{0}$.

The proof is quite easy. Indeed the curve $K_{1}$ (Figure 1b) consists of four horizontal and six vertical intervals which lie on one plane containing vectors $d_{1}$ and $d_{2}$. There are also three fragments of $K_{1}$ having three linear sections. Each such a fragment belongs to the plane parallel either to the plane containing vectors $\left(d_{1}, d_{3}\right)$ or to $\left(d_{2}, d_{3}\right)$ depending on an over-crossing or under-crossing which we have on the diagram of $K_{1}$ (Figure 1b). We can use three directions, according to the initial diameters, to move along $K_{1}$ by using reflections in planes $\Pi_{i}$.

Choosing the length of each linear part of $K_{1}$ to be equal to a multiple of the distance between $D_{j}$ and $D_{j}^{\prime}$ we get a system of equations in integers. One can check that there are many solutions and each of them gives parameters of possible coverings of $K_{1}$. The lemma is proved. Q.E.D.

The first example of a compact hyperbolic manifold fibered over $S^{1}$ is due to $\mathrm{T}$. Jorgensen $[\mathrm{J}]$; for our purposes we will use the following geometric construction which is due to W. Thurston.

We will consider a dodecahedron $D$ all of whose dihedral angles are right angles. One can represent the dodecahedron $D$ as a cube with six additional edges (Figure 2), and let $\Gamma \subset \mathrm{PSL}_{2} \mathrm{C}$ be the group generated by six isometries $\left\langle a_{1}, \ldots, a_{6}\right\rangle$ identifying opposite faces of $D$. The quotient $\mathbf{H}^{3} / \Gamma$ is an orbifold whose underlying set is a 3-torus with three embedded circles (images of the six additional edges of $D$ ) as a branching locus of order two. This orbifold admits a fibration over a circle, so there is a subgroup $\Gamma_{0} \in \Gamma$ of finite index such that the hyperbolic manifold $\mathbf{H}^{3} / \Gamma_{0}=M_{0}$ is fiber bundle over the circle see e.g. [Sul, p. 196].

Let $R$ be a reflection group determined by the faces of $D$. We have the following:

Lemma 2. The groups $\Gamma$ and $R$ have a common finite-index subgroup $R_{0}$, $\left|\Gamma: R_{0}\right|<\infty,\left|R: R_{0}\right|<\infty$.

Proof. Denote the generators of $R$ by $r_{i}, i \in\{1, \ldots, 12\}$. Now construct an epimorphism $\varphi_{1}: R \rightarrow K=\mathbf{Z}_{2}+\mathbf{Z}_{2}+\mathbf{Z}_{2}$ by mapping each of the four generators $r_{k}$ of $R$ corresponding to the top and bottom faces of $D$ to the generator $\gamma_{1}$ of the first factor of $K$; map each of the four generators $r_{j}^{\prime}$ corresponding to
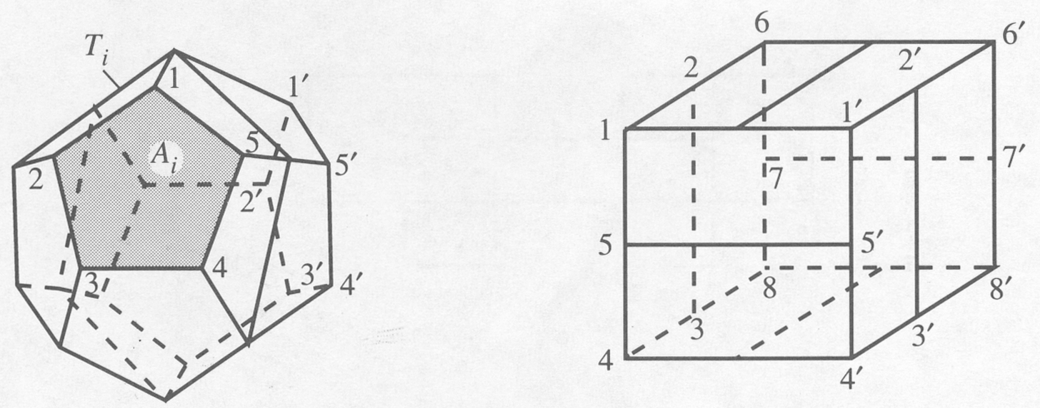

FIGURE 2 
the front and back faces to the generator $\gamma_{2}$ of the second factor and map each of the four generators $r_{l}^{\prime \prime}$ corresponding to side faces to the generator $\gamma_{3}$ of the third factor. The group $R_{0}=\operatorname{Ker} \varphi_{1}$ is generated by $r_{i} r_{j}, r_{i}^{\prime} r_{j}^{\prime}$ and $r_{i}^{\prime \prime} r_{j}^{\prime \prime} \quad(i, j \in$ $\{1,2,3,4\})$. By analogy construct an epimorphism $\varphi_{2}: \Gamma \rightarrow K$ so that the image of each pair of generators of $\Gamma$ identifying opposite faces of the "cube" $D$ is $\gamma_{i}(i=1,2,3)$. By considering the identifications on $D$ (see Figure 2 ) it is easy to see now that $\operatorname{Ker} \varphi_{2}=\operatorname{Ker} \varphi_{1}=R_{0}$, since all the generators of $\operatorname{Ker} \varphi_{1}$ belong to $\operatorname{Ker} \varphi_{2}$ and vice versa (e.g. $r_{2}^{\prime \prime} r_{1}^{\prime \prime}=a_{6}^{-1} a_{5}, r_{3}^{\prime \prime} r_{1}^{\prime \prime}=a_{5}^{2}, r_{4}^{\prime \prime} r_{2}^{\prime \prime}=$ $a_{6}^{2}, r_{4}^{\prime \prime} r_{3}^{\prime \prime}=a_{6} a_{5}^{-1}$ etc). The lemma is proved. Q.E.D.

Without loss of generality, we may assume that $\Gamma_{0}=R_{0}$ and, moreover, that $\Gamma_{0}$ is normal in $R$; for otherwise we take the intersection over all conjugacy classes $R / \Gamma_{0}$.

Now we consider the action of $R$ in both components $B_{1}$ and $B_{1}^{*}$ of $S^{3} \backslash \Sigma_{1}$, where $\Sigma_{1}=\partial B_{1}, \infty \in B_{1}^{*}$.

Suppose that $D \subset B_{1}$ and the center of $D$ coincides with the center of $B_{1}$.

Let us denote by $W$ the collection of images of geodesic planes containing faces of $D$ under elements of $R$.

A pair $\left(\rho, \rho^{\prime}\right) \subset W \times W$ will also be called opposite if the diameter of $B_{1}$ which is the common perpendicular connects the centers of $\rho$ and $\rho^{\prime}$.

We are able to consider three pairs of nonintersecting opposite geodesic planes $\left(\rho_{i}, \rho_{i}^{\prime}\right) \in W \times W$ and associate with each pair $\left(\rho_{i}, \rho_{i}^{\prime}\right)$ the pair $\left(\chi_{i}, \chi_{i}^{\prime}\right)$ of Euclidean planes such that

$$
\chi_{i} \cap B_{1}=\rho_{i} \cap B_{1}, \quad \chi_{i}^{\prime} \cap B_{1}=\rho_{i}^{\prime} \cap B_{1} .
$$

By Lemma 1 we can construct a covering of $K_{1}$ by a family of balls $\mathscr{B}=$ $\left\{B_{1}, \ldots, B_{n}\right\}$, where each $B_{i}$ is an image of $B_{i-1}$ under reflection $\tau_{i-1}$ in the plane $\Pi_{i-1}$ (see Figure 1a). Here $\left(\Pi_{1}, \Pi_{n}\right)$ is a pair of planes $\left(\chi_{i}^{\prime}, \chi_{i}\right)$ mentioned above $i \in\{1,2,3\}$.

In what follows, we will denote by $\operatorname{Stab}(A, G)$ the stabilizer of a set $A$, i.e., $\operatorname{Stab}(A, G)=\{g \in G: g A=A\}$.

The groups $L_{i}=\operatorname{Stab}\left(\chi_{i}, \Gamma_{0}\right)$ (respectively $L_{i}^{\prime}=\operatorname{Stab}\left(\chi_{i}^{\prime}, \Gamma_{0}\right)$ ) are Fuchsian groups of the first kind which are subgroups in a reflection group determined by sides of a right pentagon which is a face of $D$.

In the proof of the following lemma, we will use

Definition (see [Se2]). The group $H$ is a separable subgroup of $\Gamma$ if for each $\gamma \in \Gamma \backslash H$ there exists a subgroup $\Gamma^{*} \subset \Gamma$ of finite index such that $H \subset \Gamma^{*}$ and $\gamma \notin \Gamma^{*}$.

Notice that $\mathrm{P}$. Scott proved in [Se2] that any geometrically finite subgroup $H$ of a discrete group $\Gamma \in \mathrm{PSL}_{2} C$ is separable if $\Gamma$ is commensurable with the group $R$ generated by reflections in the faces of a compact polyhedron $P \in \mathbf{H}^{3}$ whose dihedral angles are right angles.

Also we denote by $I_{\gamma}$ an isometric sphere of an element $\gamma \in R$ and by $\mathscr{P}(\Gamma)$ an isometric fundamental domain of $\Gamma$ which is the intersection of exteriors of all isometric spheres of $\Gamma$. Let $\chi_{i}^{-}$be the half-space of $\bar{R}^{3} \backslash \chi_{i}$ not containing the center of $B_{1}$.

We will use the notation $\left[\Gamma_{0}, \Gamma_{0}\right]$ for the commutator subgroup $\Gamma_{0}$. 
Main Lemma. There exists a subgroup $\Gamma_{1} \subset \Gamma_{0}$ of finite index such that the following conditions hold:

(a) The boundary of the isometric fundamental domain $\mathscr{P}\left(\Gamma_{1}\right)$ lies in some regular $\varepsilon$-neighbourhood of $\Sigma_{1} \quad(\varepsilon>0)$.

(b) There exists three pairs of opposite planes $\left(\rho_{i}, \rho_{i}^{\prime}\right) \in W \times W$ such that $\partial \mathscr{P}\left(H_{i}\right)$ and $\partial \mathscr{P}\left(H_{i}^{\prime}\right)$ are all disjoint. Here $H_{i}=L_{i} \cap \Gamma_{1}, H_{i}^{\prime}=L_{i}^{\prime} \cap \Gamma_{1}$ $(i=1,2,3)$.

(c) There exists an element $g_{1} \in\left[\Gamma_{0}, \Gamma_{0}\right] \cap H_{1}^{\prime} \subset \Gamma_{1}$ and a plane $\pi$ orthogonal to $K_{1}$ such that $I_{g_{1}} \cap I_{g_{1}^{-1}}=\varnothing, I_{g_{1}} \cap \pi=l_{1}, I_{g_{1}^{-1}} \cap \pi=l_{2}, l_{2}=g_{1}\left(l_{1}\right)$ and $\pi \cap I_{\gamma}=\pi \cap \Sigma_{1}=\varnothing$ for each $\gamma \in \Gamma_{1} \backslash\left\{g_{1}, g_{1}^{-1}\right\}$ (see Figure 3).

(d) The group $H=\left\langle H_{1}, H_{1}^{\prime}, \ldots, H_{3}^{\prime}\right\rangle$ is a separable subgroup of $\Gamma_{1}$ and there exists a fundamental domain $R\left(\Gamma_{1}\right) \subset B_{1}^{*}$ such that $R\left(\Gamma_{1}\right) \cap\left(\chi_{i} \cup \chi_{i}^{\prime}\right)=$ $\mathscr{P}(H) \cap\left(\chi_{i} \cup \chi_{i}^{\prime}\right),(i \in\{1,2,3\})$. Z.

(e) There exists a normal finitely generated subgroup $F_{1} \triangleleft \Gamma_{1}$ such that $\Gamma_{1} / F_{1} \cong$

Proof. (a)-(b) We start by fixing some $\varepsilon$-neighbourhood $N_{1}$ of $\Sigma_{1}$. There exists at most a finite number of elements $\gamma_{i} \in \Gamma_{0} \quad(i \in I, I$ is a finite set $)$ such that $I_{\gamma_{i}} \cap \operatorname{cl}\left(B_{1}^{*} \backslash N_{1}\right) \neq \varnothing$, where $\operatorname{cl}()$ is a closure of a set.

Let us now consider nonintersecting opposite geodesic planes $\left(\rho_{i}, \rho_{i}^{\prime}\right) \in W \times$ $W$. Evidently such pairs do exist because, by reflection in opposite faces of $D$, we can get infinitely many opposite elements of $W$.

Let $N\left(\rho_{i}\right)$ (respectively $N\left(\rho_{i}^{\prime}\right)$ ) be a regular neighbourhood of $\rho_{i}$ (resp. $\rho_{i}^{\prime}$ ) in $\mathrm{cl} B_{1}$ such that $\bigcap_{i=1}^{3}\left(N\left(\rho_{i}\right) \cap N\left(\rho_{i}^{\prime}\right)\right)=\varnothing$, (where $N\left(\rho_{i}\right) \cong\{z \in \mathbf{C}:|z| \leq$ $1\} \times[0,1]$ ). Again there is at most a finite number of elements $\gamma_{j} \in L_{i}$ (resp. $\gamma_{j} \in L_{i}^{\prime}$ ) for which $I_{\gamma_{j}} \notin N\left(\rho_{i}\right)$ (resp. $I_{\gamma_{j}} \notin N\left(\rho_{i}^{\prime}\right)$ ), where $j \in J$ and $J$ is a finite set, $i \in\{1,2,3\}$.

By residual finiteness [Ma], we can choose a subgroup $\Gamma_{1}^{\prime} \subset \Gamma_{0}$ of finite index such that $\gamma_{l} \notin \Gamma_{1}^{\prime}(l \in I \cup J)$.

(c) Let us consider a plane $\pi^{\prime}$ tangent to the sphere $\Sigma_{1}$ at the point $x_{1}=$ $\Sigma_{1} \cap K_{1}$ and orthogonal to $K_{1}$ (Figure 3). We begin with the following:

Claim. There exists an element $g_{1} \in\left[L_{1}^{*}, L_{1}^{*}\right]$ such that

$$
I_{g_{1}} \cap \pi^{\prime} \neq \varnothing, \quad L_{1}^{*}=\operatorname{Stab}\left(\rho_{1}^{\prime}, \Gamma_{1}^{\prime}\right)
$$

Indeed, we can suppose that $K_{1} \cap B_{1}$ coincides with a diameter $B_{1}$ orthogonal to $\rho_{1}^{\prime}$. Take any primitive element $g_{0} \in\left[L_{1}^{*}, L_{1}^{*}\right] \subset\left[\Gamma_{0}, \Gamma_{0}\right]$ which can be represented by a simple dividing loop on the surface $\rho_{1}^{\prime} / L_{1}^{*}$. Now if (2) does not hold for $g_{0}$ we can consider a sequence $g_{n}=\xi^{n} g_{0} \xi^{-n} \quad(n \in \mathbf{Z})$ where $\xi=\tau_{\rho_{1}^{\prime}} \tau_{\rho_{1}} \in R$ (here $\tau_{\rho_{1}}$ is a reflection in $\rho_{1}$ and $\rho_{1}, \rho_{1}^{\prime}$ are opposite faces).

Evidently, $g_{n} \in\left[\xi^{n} L_{1}^{*} \xi^{-n}, \xi^{n} L_{1}^{*} \xi^{-n}\right]$.

Now we claim that $\xi^{n}\left(I_{g_{0}}\right) \cap \pi^{\prime} \neq \varnothing$ for all $n \geq N>0$. The simplest way to see this is to conjugate our action to an action in the upper-half space by an element $b \in \mathrm{PSL}_{2} \mathrm{C}$ such that $b\left(K_{1} \cap \pi^{\prime}\right)=\infty$; and so that $\gamma=b \xi b^{-1}=\lambda z$ $(\lambda \in \mathbf{R}, z \in \mathbf{C})$. The last is possible since $\xi$ is a loxodromic element having two fixed points in $K_{1} \cap \Sigma_{1}$. It is not clear that $\gamma^{n}\left(b\left(I_{g_{0}}\right)\right) \cap b\left(\pi^{\prime}\right) \neq \varnothing$ for $n \geq N$. Returning to $B_{1}$ we get the desired property.

Moreover, $\xi^{n}\left(I_{g_{0}}\right)=I_{\xi^{n}} g_{0} \xi^{-n}$, since $\xi$ preserves each plane containing $B_{1} \cap$ $K_{1}$. 


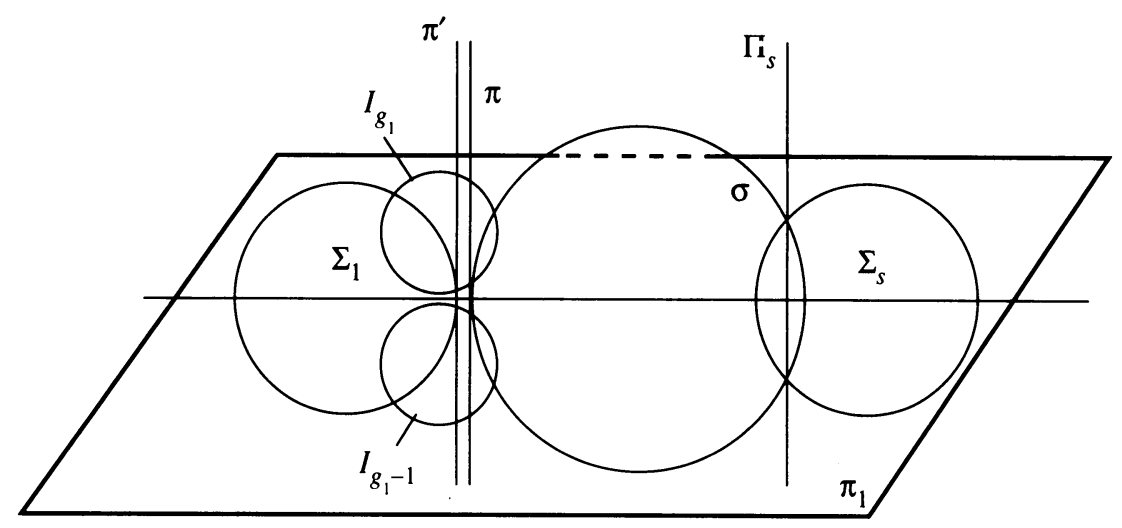

FiguRe 3

Simple assertion. If $\Gamma_{0}$ is normal in $R$ then (obviously) $\left[\Gamma_{0}, \Gamma_{0}\right]$ is normal in $R$ as well.

Clearly, we have that $g_{0} \in\left[\Gamma_{0}, \Gamma_{0}\right]$ and so is $g_{n} \in\left[\Gamma_{0}, \Gamma_{0}\right]$. Planes $\xi^{n}\left(\rho_{1}^{\prime}\right)$, $\xi^{-n}\left(\rho_{1}\right)$ are opposite and assertions (a), (b) are valid for them. Therefore, keeping the notations $L_{1}^{*}, \rho_{1}$ and $\rho_{1}^{\prime}$ for $\xi^{n} L_{1}^{*} \xi^{-n}, \xi^{-n}\left(\rho_{1}\right)$ and $\xi^{n}\left(\rho_{1}^{\prime}\right)$ we can find a primitive element $g_{1} \in\left[L_{1}^{*}, L_{1}^{*}\right]$ such that $(2)$ is valid. The claim is proved.

Now let $\pi$ be a plane parallel to $\pi^{\prime}$ such that $\pi \cap \Sigma_{1}=\varnothing, \pi \cap I_{g_{1}}=l_{1}$ and $\pi \cap I_{\left(g_{1}\right)^{n}}=\varnothing \quad(n>1)$ (Figure 3).

In what follows, we will denote by $\tau_{S}$ the reflection in a sphere $S$.

Now we claim that $\pi \cap I_{g_{1}^{-1}}=l_{2}$ and $l_{2}=g_{1}\left(l_{1}\right)$. Indeed $g_{1}=\tau_{\pi_{1}} \cdot \tau_{I_{1}}$ where $\pi_{1}$ is some plane containing $K_{1} \cap B_{1}$ and orthogonal to $\pi$ (Figure 3 ). We obtain $g_{1}\left(l_{1}\right)=g_{1}\left(I_{g_{1}} \cap \pi\right)=\tau_{\pi_{1}}\left(I_{g_{1}} \cap \pi\right)=I_{g_{1}^{-1}} \cap \pi=l_{2}$. Obviously $I_{g_{1}^{-m}} \cap \pi=\varnothing$, $m>1$.

The element $g_{1}$ which was chosen to be primitive in the group $L_{1}^{*}$ is also primitive in $\Gamma_{0}$, so $g_{1}$ generates a maximal cyclic subgroup of $\Gamma_{1}^{\prime}$. As before, there is a finite set $M \subset \mathbf{Z}$ for which $I_{g_{m}} \cap \pi \neq \varnothing, m \in M, g_{m} \in \Gamma_{1}^{\prime}$. According to separability of maximal infinite cyclic subgroups we can choose a subgroup $\Gamma_{1}^{\prime \prime} \subset \Gamma_{1}^{\prime}$ of finite index such that $\left\langle g_{1}\right\rangle \subset \Gamma_{1}^{\prime \prime}$ and $g_{m} \notin \Gamma_{1}^{\prime \prime} \quad(m \in M)$ [L].

Denote $H_{i}=\operatorname{Stab}\left(\rho_{i}, \Gamma_{1}^{\prime \prime}\right) ; H_{i}^{\prime}=\operatorname{Stab}\left(\rho_{i}^{\prime}, \Gamma_{1}^{\prime \prime}\right)$. By construction $g_{1} \in H_{1}^{\prime} \cap$ $\left[\Gamma_{0}, \Gamma_{0}\right] \subset \Gamma_{1}^{\prime \prime}$.

(d) By part (b) $H=\left\langle H_{1}, \ldots, H_{3}^{\prime}\right\rangle$ is a result of the Klein combination of $H_{i}, H_{i}^{\prime}$ and so is geometrically finite. The domain $\mathscr{P}(H)=\bigcap_{i=1}^{3} \mathscr{P}\left(H_{i}\right) \cap$ $\bigcap_{1}^{3} \mathscr{P}\left(H_{i}^{\prime}\right)$ is fundamental for $H=H_{1} * \cdots * H_{3}^{\prime}$. Let us consider a compact domain $Z=\mathscr{P}(H) \cap B_{1}^{*} \cap\left(\bigcup_{i=1}^{3} \chi_{i} \cup \bigcup_{i=1}^{3} \chi_{i}^{\prime}\right)$. Again there exists at most a finite number of elements $\gamma_{l} \in \Gamma_{1}^{\prime \prime}$ such that $\gamma_{l} Z \cap Z=\varnothing, l \in L$.

The group $H$ is a geometrically finite subgroup of $\Gamma_{1}^{\prime \prime}$ which is commensurable with a reflection group in a right angled polyhedron, so by the theorem of P. Scott [Sc2] mentioned above we can state that $H$ is a separable subgroup of $\Gamma_{1}^{\prime \prime}$. So, there is a subgroup $\Gamma_{1} \subset \Gamma_{1}^{\prime \prime}$ of finite index for which $H \subset \Gamma_{1}$ and $\gamma_{l} \notin \Gamma_{1}$ for all $l \in L$. Evidently, $g_{1} \in H \subset \Gamma_{1}$ and the assertions of all previous steps are valid for $\Gamma_{1}$ as well. 
Set $A=\bigcup_{i=1}^{3}\left(\chi_{i} \cup \chi_{i}^{\prime}\right)$; if $\gamma A \cap A \neq \varnothing$ for $\gamma \in \Gamma_{1} \backslash H$ then $\gamma_{0} h_{1} Z \cap h_{2} Z \neq \varnothing$, $h_{i} \in H$, which is impossible.

Moreover, due to compactness of surfaces $\chi_{i} / H_{i}$ and $\chi_{i}^{\prime} / H_{i}^{\prime}$, we can choose regular neighbourhoods $V_{i}$ and $V_{i}^{\prime}$ of $\chi_{i} \cap B_{i}^{*}$ and $\chi_{i}^{\prime} \cap B_{1}^{*}$ such that $H_{i} V_{i}=V_{i}$, $H_{i}^{\prime} V_{i}^{\prime}=V_{i}^{\prime}$ and $\gamma V \cap V=\varnothing$ for $V=\bigcup_{i=1}^{3}\left(V_{i} \cup V_{i}^{\prime}\right), \gamma \in\left(\Gamma_{1} \backslash H\right)$.

The domain $W=\mathscr{P}\left(\Gamma_{1}\right) \cap\left(B_{1}^{*} \backslash \Gamma_{1} V\right)$ is fundamental for the action of $\Gamma_{1}$ on $B_{1}^{*} \backslash \Gamma_{1} V$ and evidently we get the required domain $R\left(\Gamma_{1}\right)=W \cup(\mathscr{P}(H) \cap \mathrm{cl} V)$.

(e) The group $\Gamma_{1}$ is a finite-index subgroup of $\Gamma_{0}$ and therefore the group $F_{1}=F_{0} \cap \Gamma_{1}$ is a finitely generated normal subgroup in $\Gamma_{1}$. We observe that $g_{1} \in\left[\Gamma_{0}, \Gamma_{0}\right] \cap H_{1}^{\prime} \subset F_{0} \cap H_{1}^{\prime} \subset F_{1} \cap H_{1}^{\prime}$, since $\left[\Gamma_{0}, \Gamma_{0}\right] \subset F_{0}$. The lemma is proved. Q.E.D.

Let us introduce some terminology and notation which we will use later.

Suppose $\Gamma_{i}(i=1,2)$ are Kleinian groups in $M(3), U=\Gamma_{1} \cap \Gamma_{2}$ is a common subgroup and there exists a compact surface $D \subset S^{3}$ such that $S^{3} \backslash D=B_{1} \cup B_{2}, D \cap \Omega(U) \subset \Omega_{\Gamma_{1}} \cap \Omega_{\Gamma_{2}}$.

We will say that $D$ is strongly invariant under $U$ in $\Gamma_{i}$ iff $g\left(B_{j}\right) \subset B_{i}$, $g D \cap D=\varnothing, h D=D, g \in \Gamma_{i} \backslash U, h \in U, i \neq j, i, j \in\{1,2\}$.

It follows from Maskit's Combination Theorem that, if (int $D$ ) $/ U$ is a compact surface in $\Omega_{\Gamma_{i}} / \Gamma_{i}$ and $D$ is strongly invariant under $U$ in $\Gamma_{i}$, then the group $\Gamma=\left\langle\Gamma_{1}, \Gamma_{2}\right\rangle$ is discontinuous and isomorphic to $\Gamma=\Gamma_{1} *_{U} \Gamma_{2}$ [M1, M2].

\section{Proof of Theorems A AND B}

Recall that we have a trefoil represented by piecewise linear curve $K_{1} \subset S^{3}$ and the covering $\mathscr{B}_{1}=\left\{B_{1}, \ldots, B_{n}\right\}$ of $K_{1}$ by balls centered on $K_{1}$. Let us denote by $\Lambda_{i}=B_{i} \cap B_{i+1} \cap \Pi_{i}$. Now we change this construction in the following way. According to the Main Lemma, we can find a sphere $\sigma \subset S^{3}$ centered on $K_{1}$ such that $\sigma \subset \pi^{-}, \sigma \cap I_{g_{1}}=l_{1}$ and $\sigma \cap \Sigma_{s}=\Lambda_{s}$ for some $s<n$ (see Figure 3), where $\pi^{-}$is the right half space of $S^{3} \backslash \pi$.

Moreover (without loss of generality, due to the slight moving of $\sigma$ ) we can suppose that the angle between $\sigma$ and $I_{g_{1}}$ is equal to $\pi /(2 n), n \in \mathbf{Z}$. Simple geometric considerations show that $\sigma$ can be chosen in such a way that $\sigma \cap \Sigma_{1}=\varnothing$; namely this is true for sufficiently large radius $\sigma$ and lengths of linear sections $K_{1}$.

By assertion (c) of the Main Lemma, it follows that $\sigma \cap I_{g_{1}^{-1}}=l_{2}=g_{1}\left(l_{1}\right)$ and $\sigma \cap I_{\gamma}=\varnothing, \gamma \in \Gamma_{1} \backslash\left\{g_{1}, g_{1}^{-1}\right\}$.

Let $K=K_{1} \# \tau_{\sigma}\left(K_{1}\right)$ be a double knot, i.e. a connected sum of two trefoils along $\sigma$ (Figure $1 \mathrm{~b}$ ). Consider the the family of balls $\mathscr{B}=\mathscr{B}_{1}^{\prime} \cup \mathscr{B}_{2}^{\prime}$ where $\mathscr{B}_{1}^{\prime}=\left(B_{1}, \ldots, B_{s}\right), \mathscr{B}_{2}^{\prime}=\tau_{\sigma}\left(\mathscr{B}_{1}^{\prime}\right)$.

Let $\Gamma_{k+1}=\tau_{k} \Gamma_{k} \tau_{k}, B_{k+1}^{*}=\tau_{k}\left(B_{k}^{*}\right)(k=1, \ldots, s-1)$ and let $\partial \mathscr{B}_{1}$ denote the boundary $\partial\left(\bigcap_{k=1}^{s-1} \tau_{k}\left(B_{k}^{*}\right)\right)$.

Consider the plane $\Pi_{1}=\chi_{1}$ and group $\Gamma_{1}^{\prime}=\operatorname{Stab}\left(\left(B_{1}^{*} \backslash \Gamma_{1} \Pi_{1}^{-}\right), \Gamma_{1}\right)$ where $\Pi_{1}^{-}$is a component of $B_{1}^{*} \backslash \Pi_{1}$ left invariant under $\operatorname{Stab}\left(\Pi_{r}, \Gamma_{r}\right)$.

Lemma 3. There exists a subgroup $\Gamma_{i}^{\prime} \subset \Gamma_{i}$ isomorphic to the fundamental group of a compact hyperbolic 3-manifold with geodesic boundary such that the following hold. 
1. Group $G_{s}=\left\langle\Gamma_{1}^{\prime}, \ldots, \Gamma_{s}^{\prime}\right\rangle$ is a discontinuous group acting freely and cocompactly on a component $\Omega_{G_{s}} \subset S^{3} \quad\left(\infty \in \Omega_{G_{s}}\right)$.

2. The group $G_{s}$ is a result of the Maskit combination of groups $\Gamma_{s}^{\prime}$ and $G_{s-1}^{\prime}$ along the sphere $\Pi_{s} \cup\{\infty\} \quad\left(G_{1}^{\prime}=\Gamma_{1}^{\prime}\right.$ and $G_{s-1}^{\prime}$ is to be defined inductively).

3. There exists a fundamental domain $R\left(G_{s}\right)$ such that $\partial R\left(G_{s}\right)$ is isotopic to $\partial \mathscr{B}_{1}$.

4. There exists a nontrivial finitely generated normal subgroup $F_{s} \triangleleft G_{s}^{*}$ in a subgroup $G_{s}^{*}$ of $G_{s}$ and $\left|G_{s}: G_{s}^{*}\right|<\infty$.

Proof. Let us prove this lemma by induction on $k$. For $k=1$ the assertion is true, namely we set $G_{1}=\Gamma_{1}$ and all conclusions now follow from the Main Lemma.

Now we suppose that it is true for $k<r, 2 \leq r \leq s$, i.e. the group $G_{r-1}=$ $\left\langle\Gamma_{1}^{\prime}, \ldots, \Gamma_{r-1}^{\prime}\right\rangle$ is discontinuous and has the fundamental domain $R\left(G_{r-1}\right)$ such that $R\left(G_{r-1}\right) \cap \Pi_{r-1}$ is the fundamental domain for the action of $\mathscr{H}_{r-1}=$ $\operatorname{Stab}\left(\Pi_{r-1}, \Gamma_{r-1}^{\prime}\right)$ on $\Pi_{r-1}$.

By construction the plane $\Pi_{r-1}$ is the image of one of the planes $\chi_{i}, \chi_{i}^{\prime}$ under composition $\tau_{r-2} \cdots \tau_{1}$ and the group $\mathscr{H}_{r-1}=\operatorname{Stab}\left(\Pi_{r-1}, G_{r-1}\right)$ is conjugate to one of $H_{i}$ or $H_{i}^{\prime} \quad(i=1,2,3)$ (see Main Lemma). Now we have to define groups $G_{r-1}^{\prime}$ and $\Gamma_{r}^{\prime}$ which take part in the process of combination.

By the remark above, there is a component $\Pi_{r-1}^{-}$of $B_{r-1}^{*} \backslash \Pi_{r-1}$ which is strongly invariant under $\mathscr{H}_{r-1}$ in $G_{r-1}$. Denote $\Pi_{r-1}^{+}=\tau_{r-1}\left(\Pi_{r-1}^{-}\right)$. Consider the domains $\Omega_{G_{r-1}}^{-}=\Omega_{G_{r-1}} \backslash G_{r-1}\left(\Pi_{r-1}^{-}\right)$and $B_{r}^{-}=B_{r}^{*} \backslash \Gamma_{r}\left(\Pi_{r-1}^{+}\right)$and the groups $G_{r-1}^{\prime}=\operatorname{Stab}\left(\Omega_{G_{r-1}}^{-}, G_{r-1}\right), \Gamma_{r}^{\prime}=\operatorname{Stab}\left(B_{r}^{-}, \Gamma_{r}\right)$. Evidently, $\mathscr{H}_{r-1}=G_{r-1} \cap \Gamma_{r}^{\prime}$.

Claim 1. All conditions of Maskit's Combination Theorem are satisfied for $\left\langle G_{r-1}^{\prime}, \Gamma_{r}^{\prime}\right\rangle=G_{r}$.

Proof of the claim. Consider $\delta_{r-1}=R\left(G_{r-1}\right) \cap \Pi_{r-1}$ which is the boundary of the fundamental domain $\mathscr{P}\left(\mathscr{H}_{r-1}\right) \cap \Pi_{r-1}$ for the action on $\Pi_{r-1}$. By construction $\operatorname{cl}\left(R\left(\Gamma_{r}\right)\right)$ is homeomorphic to a ball, so $\delta_{r-1}$ bounds an embedded disk $V_{r-1} \subset$ $\Pi_{r-1}^{-} \cap R\left(\Gamma_{r}\right)$. Let $U_{r-1}=\bigcup_{h \in \mathscr{K}_{r-1}} h V_{r-1}$, which is a surface strongly invariant under $\mathscr{H}_{r-1}$ in the groups $G_{r-1}^{\prime}$ and $\Gamma_{r}^{\prime}$. Moreover $U_{r-1} \subset \Omega_{G_{r-1}^{\prime}} \cap B_{r}^{*}$ and $U_{r-1} / \mathscr{H}_{r-1}$ is homeomorphic to a $\Pi_{r-1} / \mathscr{H}_{r-1}$.

Now we have to prove that $g\left(\operatorname{cl}\left(U_{r-1}\right)\right) \cap \operatorname{cl}\left(U_{r-1}\right)=\varnothing, g \in\left(G_{r-1}^{\prime} \cup \Gamma_{r}^{\prime}\right) \backslash \mathscr{H}_{r-1}$. The last will follow from $g\left(\operatorname{cl}\left(\Pi_{r-1}^{-}\right)\right) \cap \mathrm{cl} \Pi_{r-1}^{-}=\varnothing$. Suppose first that there exists $\gamma_{0} \in G_{r-1}^{\prime} \backslash \mathscr{H}_{r-1}$ such that $\gamma_{0}\left(\Pi_{r-1}^{-}\right) \cap \Pi_{r-1}^{-} \neq \varnothing$. Let us show that $\gamma_{0} \in$ $\Gamma_{r-1}^{\prime}$, indeed there exist elements $h_{i} \in \Gamma_{r-1}^{\prime}$ such that $h_{1} \gamma_{0} h_{2}\left(\operatorname{int}\left(R\left(G_{r-1}^{\prime}\right)\right)\right) \cap$ $\operatorname{int}\left(R\left(G_{r-1}^{\prime}\right)\right) \neq \varnothing$. Hence, $\gamma_{0} \in\left(\Gamma_{r-1}^{\prime} \cap G_{r-1}^{\prime}\right)=\mathscr{H}_{r-1}$ what is impossible. The same arguments work when $g \in \Gamma_{r}^{\prime} \backslash \mathscr{H}_{r-1}$.

Suppose now that $\gamma_{0}\left(\operatorname{cl}\left(\Pi_{r-1}^{-}\right)\right) \cap \operatorname{cl}\left(\Pi_{r-1}^{-}\right)=\left\{x_{0}\right\} \in \Lambda\left(\mathscr{H}_{r}\right)$ and consider the action of $\Gamma_{r-1}^{\prime}$ on a sphere $\Sigma_{r-1} .^{2}$

Let $w_{1}$ be a component of $\Sigma_{r-1} \backslash \Lambda\left(\mathscr{H}_{r-1}\right)$ such that $w_{1} \subset \Pi_{r-1}^{-}$. We get that $w_{1} \cap w_{2}=\left\{x_{0}\right\} \in \Lambda\left(\mathscr{H}_{r-1}\right)$ where $w_{2}=\gamma_{0}\left(w_{1}\right)$.

Group $\mathscr{H}_{r-1}$ is Fuchsian, so each limit point of $\mathscr{H}_{r-1}$ is an approximation point [Be-M]. Now consider a model of the hyperbolic plane in $\Sigma_{r-1} \backslash \omega_{1}$. There exists a geodesic $l$ with end points $\left\{x_{0}, y_{0}\right\} \in \Lambda\left(\mathscr{H}_{r-1}\right)$ such that for

\footnotetext{
${ }^{2}$ These considerations concluding the proof of Claim 1 are suggested by M. Kapovich.
} 
some sequence $\left\{h_{n}\right\} \in \mathscr{H}_{r-1}$ we have $h_{n} l \cap T \neq \varnothing$ for a compact subset $T \subset$ $\left(\Sigma_{r-1} \backslash \omega_{1}\right)$. Evidently, $h_{n}\left(\omega_{2}\right) \cap h_{n_{0}}\left(\omega_{2}\right) \neq \varnothing \quad\left(n \geq n_{0}\right)$ because

$$
\lim _{n \rightarrow \infty} h_{n} \omega_{2}=\Sigma_{1} \backslash w_{1} \text {. }
$$

We have deduced that $\gamma_{0}^{-1} h_{n_{0}}^{-1} h_{n} \gamma_{0}\left(\Pi_{r-1}^{-}\right) \cap \Pi_{r-1}^{-} \neq \varnothing$ which is impossible. The same arguments show that $\gamma\left(\operatorname{cl}\left(\Pi_{r-1}^{+}\right)\right) \cap \operatorname{cl}\left(\Pi_{r-1}^{+}\right)=\varnothing, \gamma \in \Gamma_{r}^{\prime} \backslash \mathscr{H}_{r-1}$. Claim 1 is proved.

By Maskit's Combination Theorem we have

$$
G_{r}=G_{r-1}^{\prime} * \mathscr{H}_{r-1} \Gamma_{r}^{\prime}
$$

To prove assertion 3 of the lemma, we notice that the domain $R\left(\Gamma_{r}^{\prime}\right)=$ $\tau_{r-1} \cdots \tau_{1}\left(R\left(\Gamma_{1}^{\prime}\right)\right)$ (here $\left.R\left(\Gamma_{1}^{\prime}\right)=R\left(\Gamma_{1}\right) \cap B_{1}^{-}\right)$is fundamental for the action of $\Gamma_{r}^{\prime}$ on $B_{r}^{-}$which follows from part (d) of the Main Lemma. There exists a neighbourhood $V_{r-1}$ of $\Pi_{r-1} \cap B_{r}^{*}$ such that $R\left(G_{r-1}\right) \cap V_{r-1}=R\left(\Gamma_{r}^{\prime}\right) \cap V_{r-1}=$ $\mathscr{P}\left(\mathscr{H}_{r-1}\right) \cap V_{r-1}$ (see proof of part (d) of the Main Lemma). Evidently, domain $R\left(G_{r-1}^{\prime}\right)=R\left(G_{r-1}\right) \cap \Omega_{G_{r-1}}^{-}$is fundamental for the action of $G_{r-1}^{\prime}$ on $\Omega_{G_{r-1}}^{-}$. Again using Maskit's Combination Theorem, we get that $R\left(G_{r}\right)=R\left(G_{r-1}^{\prime}\right) \cap$ $R\left(\Gamma_{r}^{\prime}\right)$ is a fundamental domain for $G_{r}$. Obviously, $\delta_{r}=R\left(G_{r}\right) \cap \Pi_{r}$ bounds a fundamental domain for $\mathscr{H}_{r}$ on $\Pi_{r}$ and $\partial R\left(G_{r}\right)$ is isotopic to $\partial\left(\bigcap_{k=1}^{r} \tau_{k}\left(B_{k}^{*}\right)\right)$.

The assertion 4 of the present lemma will follow from the following.

Claim 2. The group $G_{r}$ is isomorphic to a subgroup of $R \subset \operatorname{Isom}\left(\mathbf{H}^{3}\right)$ of finite index.

Proof. First of all we prove that there exists a monomorphism $i_{r}: G_{r} \rightarrow R$. Let us suppose that it is true for $k \leq(r-1)$. The group $\Gamma_{1}$ has a fundamental domain $R\left(\Gamma_{1}\right) \subset \mathbf{H}^{3}$ such that $R\left(\Gamma_{1}\right) \cap \Pi_{1}$ is a fundamental one for the action of $\mathscr{H}_{1}$ on $\Pi_{1}^{-}$. Remember that the first combination was along the plane $\Pi_{1}$ and that there exists a geodesic plane $\xi_{1}=\rho_{1}^{\prime} \in W$ for which $\Pi_{1} \cap \Sigma_{1}=\partial \xi_{1}$ where $H_{1}=\operatorname{Stab}\left(\Pi_{1}, \Gamma_{1}^{\prime}\right)=\operatorname{Stab}\left(\xi_{1}, \Gamma_{1}^{\prime}\right)$ (see Main Lemma).

Consider group $\widetilde{G}_{2}=\left\langle\Gamma_{1}^{\prime}, \widetilde{\Gamma}_{2}\right\rangle$ where $\widetilde{\Gamma}_{2}=\tau_{\xi_{1}} \Gamma_{1}^{\prime} \tau_{\xi_{1}}$ and $\tau_{\xi_{1}}$ is a reflection in $\xi_{1}$. Obviously, there exists an isomorphism $i_{2}: G_{2} \rightarrow \widetilde{G}_{2}$. In fact, $G_{2}$ is a result of a bending deformation of $\widetilde{G}_{2}$ along $\xi_{1}$ [T1].

Recall that $\tau_{i}$ means reflection in the plane $\Pi_{i}$. We will repeat our construction of $G_{3}$ by modelling it in $\mathbf{H}^{3}$. There is a subgroup $\widetilde{\mathscr{H}}_{2}$ isomorphic to $\mathscr{H}_{2}$ which stabilizes the plane $\xi_{2}=\tau_{\xi_{1}}\left(\rho_{1}\right) \in W$. Let us consider groups $\widetilde{\Gamma}_{3}=\tau_{\xi_{2}} i_{2}\left(\tau_{2} \Gamma_{3}^{\prime} \tau_{2}\right) \tau_{\xi_{2}}$ and $\widetilde{G}_{3}=i_{2}\left(G_{2}^{\prime}\right) * \widetilde{\mathscr{H}}_{2} \widetilde{\Gamma}_{3}$. Evidently we have an isomorphism $i_{3}: G_{3} \rightarrow \widetilde{G}_{3}$.

Let us suppose that isomorphisms $i_{r-1}: G_{r-1} \rightarrow \widetilde{G}_{r-1}$ have been constructed $(r \leq s)$.

There exists a geodesic plane $\xi_{r-1} \subset W \subset B_{1}$ which is invariant under $\widetilde{\mathscr{H}}_{r-1}=\operatorname{Stab}\left(\xi_{r-1}, \widetilde{G}_{r-1}\right)$. Of course, by induction $\xi_{r-1}=\tau_{\xi_{r-2}}\left(\xi_{r-3}\right)$.

Let $\widetilde{\Gamma}_{r}=\tau_{\xi_{r-1}}\left(i_{r-1}\left(\tau_{r-1} \Gamma_{r}^{\prime} \tau_{r-1}\right)\right) \tau_{\xi_{r-1}}$ and $\widetilde{G}_{r}=\left\langle i_{r-1}\left(G_{r-1}^{\prime}\right), \widetilde{\Gamma}_{r}\right\rangle$.

Take a fundamental domain $R\left(\widetilde{G}_{r-1}\right)$ such that $R\left(\widetilde{G}_{r-1}\right) \cap \xi_{r-1}$ is a fundamental domain for $\widetilde{\mathscr{H}}_{r-1}$. Planes in $W$ are all disjoint, so by using the Maskit Combination Theorem we get that $\widetilde{G}_{r}=i_{r-1}\left(G_{r-1}^{\prime}\right) * \widetilde{\mathscr{H}}_{r-1} \widetilde{\Gamma}_{r}$. An isomorphism $i_{r}: G_{r} \rightarrow \widetilde{G}_{r}$ have been constructed. 
Now it remains to prove that $\Gamma_{0}$ and $\widetilde{G}_{r}$ are commensurable. Let us notice that $\mathbf{H}^{3} / \widetilde{G}_{r}$ is compact since $R\left(\widetilde{G}_{r}\right)$ is a compact subset of $\mathbf{H}^{3}=B_{1}$. By the construction index $\left|R: \Gamma_{0}\right|<\infty$ and we have that the index $\left|\widetilde{G}_{r}:\left(\Gamma_{0} \cap \widetilde{G}_{r}\right)\right|$ is also finite, hence the manifold $\mathbf{H}^{3} /\left(\Gamma_{0} \cap \widetilde{G}_{r}\right)$ is compact. Now it follows that the covering $\mathbf{H}^{3} /\left(\Gamma_{0} \cap \widetilde{G}_{r}\right) \rightarrow \mathbf{H}^{3} / \Gamma_{0}$ is finite and the groups $\widetilde{G}_{r}$ and $\Gamma_{0}$ are commensurable.

There is a finitely generated normal subgroup $F_{0} \triangleleft \Gamma_{0}$ and therefore $i_{s}^{-1}\left(F_{0} \cap \widetilde{G}_{s}\right)=F_{s}$ is a normal finitely generated subgroup of $i_{s}^{-1}\left(\Gamma_{0} \cap \widetilde{G}_{s}\right)^{\prime}=$ $G_{s}^{*}$, which is a finite index subgroup of $G_{s}$. Lemma 3 is proved. Q.E.D.

We shall denote by $\operatorname{int}(\sigma)$ the bounded component $S^{3} \backslash \sigma$. Recall that we have shown that group $G_{s}$ is discontinuous and $G_{s}$ is the result of Maskit's combination of groups acting in hyperbolic 3-space. In fact, one can equivalently think of $G_{s}$ as the result of bending deformation in initial group $\Gamma_{1} \subset$ Iso $\left(\mathbf{H}^{3}\right)$ along the family of embedded totally geodesic planes constructed in the Main Lemma [T1].

Now we wish to emphasize that the domain of discontinuity $\Omega\left(G_{s}\right)$ consists of two invariant components $\Omega_{G_{s}}$ and $\Omega_{G_{s}}^{\prime}\left(\infty \in \Omega_{G_{s}}\right)$. To see that, one can use Tukia's theorem [Tu] stating that the limit set $\Lambda\left(G_{s}\right)$ is homeomorphic to the limit set of the group $\widetilde{G}_{s}$ (which is a sphere $S^{2}$ embedded in $S^{3}$ ) isomorphic to $G_{s}$ and which was constructed in the Claim 2 of Lemma 3. Thus, $\Lambda\left(G_{s}\right)$ divides $S^{3}$ into two invariant components $\Omega_{G_{s}}$ and $\Omega_{G_{s}}^{\prime}$.

Remark. Although we will not use this fact later one can also prove that each invariant component of $G_{s}$ is simply connected. For example, let us show this for the component $\Omega_{G_{s}}$. We see that the groups $\pi_{1}\left(M_{G_{s}}=\Omega_{G_{s}} / G_{s}\right)$ and $G_{s}$ are isomorphic (one needs to express the first group as a free product with amalgamation by Van Kampen theorem and the analogous expression can be obtained for the second group by Maskit's combination theorem). Further, we have an epimorphism $\tau: \pi_{1}\left(M_{G_{s}}\right) \rightarrow G_{s}$ induced by the covering projection and since $G_{s}$ is Hopfian $\left(G_{s}\right.$ is residually finite as a subgroup of $M(3)$ ), it follows that $\Omega_{G_{s}}$ is simply connected. The proof is analogous for the component $\Omega_{G_{s}}^{\prime}$.

Consider now a fundamental domain $R^{\prime}\left(G_{s}\right)$ for the action of $G_{s}$ on the component $\Omega_{G_{s}}^{\prime}$, constructed in exactly the same way as we did for $R\left(G_{s}\right)$.

By our construction (see the beginning of $\S 4$ ) we have that $\sigma \cap R\left(G_{s}\right)=$ $\left(\mathscr{P}\left(H_{s}\right) \cap \sigma \cap R\left(G_{s}\right)\right) \backslash\left(I_{g_{1}} \cup I_{g_{1}^{-1}}\right)$ and $\sigma \cap R^{\prime}\left(G_{s}\right)=\mathscr{P}\left(H_{s}\right) \cap \sigma \cap R^{\prime}\left(G_{s}\right)$.

We shall identify $\mathrm{H}^{4}$ with the 4-ball $\mathrm{B}^{4}$ endowed with the Poincare metric and we identify the boundary $\partial \mathbf{H}^{4}$ with the 3 -sphere $S^{3}$ where all our groups act.

Now construct a 4-dimensional fundamental polyhedron $\mathscr{R}\left(G_{s}\right) \subset \mathbf{H}^{4} \cup$ $\Omega\left(G_{s}\right)$ by extending each sphere whose part is a face of $R\left(G_{s}\right)$ to a geodesic hypersurface in $\mathbf{H}^{4}$ orthogonal to $\partial \mathbf{H}^{4}$. Our polyhedron $\mathscr{R}\left(G_{s}\right)$ consists of points lying in the closure of the exterior of every one of these geodesic hypersurfaces. we see that $\mathscr{R}\left(G_{s}\right) \cap \Omega\left(G_{s}\right)$ has two components $R\left(G_{s}\right)$ and $R^{\prime}\left(G_{s}\right)$ which form the fundamental domain for the action of the group $G_{s}$ in $S^{3}$ (this gives an independent way of getting $\left.R^{\prime}\left(G_{s}\right)\right)$.

We still have $\mathscr{R}\left(G_{s}\right) \cap \tilde{\sigma}=\left(\widetilde{\mathscr{P}}\left(H_{s}\right) \cap \tilde{\sigma}\right) \backslash\left(\widetilde{I}_{g_{1}} \cap \widetilde{I}_{g_{1}^{-1}}\right)$ (here we use the notation $\tilde{A}$ to denote the geodesic extension of a set $A$ to $\mathbf{H}^{4}$ ). Indeed, if it were not 
so, then there would exist a 3 -face $\tilde{f} \in \partial \mathscr{R}\left(G_{s}\right)$ such that $\tilde{f} \cap \tilde{\sigma} \neq \varnothing$ and $\tilde{f}$ does not belong to $\widetilde{I}_{g_{1}} \cup \widetilde{I}_{g_{1}^{-1}} \cup \partial \widetilde{\mathscr{P}}\left(H_{s}\right)$. Then projecting on $\partial \mathbf{H}^{4}$ we obtain $f \cap \sigma \neq \varnothing$ and $f \notin\left(I_{g_{1}} \cup I_{g_{1}^{-1}} \cup \partial \mathscr{P}\left(H_{s}\right)\right)$ which is impossible by Main Lemma.

Now we are going to construct a bigger group $G$ which will be our final group (modulo a finite-index subgroup). Set $\mathscr{R}^{-}\left(G_{s}\right)$ to be the closure of the set $\mathscr{R}\left(G_{s}\right) \cap \operatorname{ext}(\tilde{\sigma})$ where $\operatorname{ext}(\tilde{\sigma})$ is a half-space $\mathbf{H}^{4} \backslash \tilde{\sigma}$ containing the origin. Now consider the polyhedron

$$
\mathscr{R}=\mathscr{R}^{-}\left(G_{s}\right) \cap \tau_{\tilde{\sigma}}\left(\mathscr{R}^{-}\left(G_{s}\right)\right)
$$

where $\tau_{\tilde{\sigma}}$ is the reflection in $\tilde{\sigma}$.

We claim that faces of $\mathscr{R}$ are paired by transformations of the group Iso $\left(\mathbf{H}^{4}\right)$. Recall that $\tilde{\sigma} \cap \widetilde{\mathscr{P}}\left(H_{s}\right)$ is a fundamental domain for the action of $H_{s}$ on $\tilde{\sigma}$. Since $\tilde{\sigma}$ contains the limit set of $H_{s}$ all isometric spheres bounding $\widetilde{\mathscr{P}}\left(H_{s}\right)$ are orthogonal to $\tilde{\sigma}$. Hence, they are invariant under the reflection $\tau_{\tilde{\sigma}}$. From the formula $\partial \mathscr{R} \cap \tilde{\sigma}=\tilde{\sigma} \cap\left(\partial \widetilde{\mathscr{P}}\left(H_{s}\right) \cup \widetilde{I}_{g_{1}} \cup \widetilde{I}_{g_{1}^{-1}}\right)$, it follows that the only edges of $\mathscr{R}$ lying on $\tilde{\sigma}$ are the edges $\tilde{l}_{1}=\mathscr{R} \cap \widetilde{I}_{g_{1}}, \tilde{l}_{2}=\mathscr{R} \cap I_{g_{1}^{-1}}$. There is a pairing of faces of $\mathscr{R}^{-}\left(G_{s}\right)$ by a system $\left\langle g_{1}, \gamma_{1}, \ldots, \gamma_{l}\right\rangle$ of elements generating $G_{s}$ which comes from the pairing of faces of the fundamental polyhedron $\mathscr{R}\left(G_{s}\right)$ [M2, p. 69]. Let us now consider the set of elements $S=$ $\left\langle g_{1}, \gamma_{1}, \ldots, \gamma_{l}, g_{2}, \gamma_{1}^{\prime}, \ldots, \gamma_{l}^{\prime}\right\rangle$, where $\gamma_{i}^{\prime}=\gamma_{i}^{\tau_{\sigma}}, g_{2}=g_{1}^{\tau_{\sigma}}$. The above means that $S$ forms a complete collection of face-pairing transformations of the polyhedron $\mathscr{R}$. Indeed, all sides of $\partial \mathscr{R}$ are either those of $\partial \mathscr{R}^{-}\left(G_{s}\right)$ or belong to $\tau_{\tilde{\sigma}}\left(\partial \mathscr{R}^{-}\left(G_{s}\right)\right)$ and, so are paired by elements of $S$.

Let $G$ be a group generated by the system $S$. Our goal is to prove that $\mathscr{R}$ is a fundamental polyhedron for $G$ and hence $G$ is discontinuous. By Poincaré's Polyhedron Theorem we have only to check that the sum of angles of each cycle of edges is $2 \pi / q$ where $q$ is the order of the generator of the stabilizer of the edge [M2, p. 69].

An arbitrary cycle in $\partial \mathscr{R}$ either consists of edges in $\partial\left(\mathscr{R}^{-}\left(G_{s}\right)\right)$ and $\partial\left(\tau_{\tilde{\sigma}}\left(\mathscr{R}^{-}\left(G_{s}\right)\right)\right)$ or is a cycle consisting of faces $\tilde{\zeta}_{i}, \tilde{\zeta}_{i}^{\prime}$ lying on isometry spheres $\tilde{I}_{g_{i}}, \widetilde{I}_{g_{i}^{-1}}(i=1,2)$. If now $C=\left(e_{1}, \ldots, e_{t}\right)$ is a cycle of the first type then the sum of the angles of $C$ satisfies the condition above, since $C$ is also a cycle of edges of one of the fundamental polyhedrons $\mathscr{R}\left(G_{s}\right)$ or $\tau_{\tilde{\sigma}}\left(\mathscr{R}\left(G_{s}\right)\right)$. It remains to verify this condition for the cycle of the second type.

Claim. All conditions of Poincaré's Polyhedron Theorem are valid at the edges $\tilde{l}_{i}=\tilde{\zeta}_{i} \cap \tilde{\zeta}_{i}^{\prime}(i=1,2)$.

Proof. We will check that $\tilde{l}_{1}$ (respectively $\tilde{l}_{2}$ ) is the fixed axis of elliptic element $g=g_{1}^{-1} g_{2}$ (resp. $g_{2} g_{1}^{-1}$ ) of order $n$. Obviously one can check this by considering the action of $g$ in $S^{3}$.

Let us first prove that $\left.g\right|_{l_{1}}=\left.g_{1}^{-1} g_{2}\right|_{l_{1}}=\mathrm{id}$. Indeed we have that $g_{1}=i_{\pi_{1}} \cdot i_{I_{g_{1}}}$, where $i_{S}$ denotes reflection in a sphere $S$ (see Figure 3 ).

Using the fact that $\sigma$ meets $\pi_{1}$ at right angles, a calculation gives us $g=$ $\left(i_{I_{g_{1}}} \cdot \tau_{\sigma}\right)^{2}$ and $\left.g\right|_{l_{1}}=$ id because both $\left.\tau_{\sigma}\right|_{\sigma}=\mathrm{id}$ and $\left.i_{I_{g_{1}}}\right|_{l_{1}}=\mathrm{id}$.

Also, we remember that the angle $\alpha\left(\zeta_{1}, \zeta_{2}\right)=\alpha\left(\tilde{\zeta}_{1}, \tilde{\zeta}_{2}\right)=\alpha\left(\zeta_{1}^{\prime}, \zeta_{2}^{\prime}\right)=\pi / n$ (see the beginning of $\S 4$ ), hence $\left(i_{I_{g_{1}}} \tau_{\sigma}\right)^{2 n}=1$, so $g^{n}=1$. The claim is proved. Q.E.D. 
We have now proved the following.

Lemma 4. The polyhedron $\mathscr{R}$ is a fundamental polyhedron for the group $G$ acting in $\mathbf{H}^{4}$.

Corollary 4.1. The group $G$ acts discontinuously in $S^{3}$ and has a fundamental domain which consists of two connected components $R(G)$ and $R^{\prime}(G)$. Generators of $G$ identify faces of $R(G)$ with faces of $R(G)$ and faces of $R^{\prime}(G)$ with faces of $R^{\prime}(G)$.

Proof. From the construction of $\mathscr{R}$ it is easy to see that $\mathscr{R} \cap \Omega(G)$ consists of two components $R(G)=R\left(G_{s}\right) \cap \tau_{\sigma}\left(R\left(G_{s}\right)\right)$ and $R^{\prime}(G)=R^{\prime}\left(G_{s}\right) \cap \tau_{\sigma}\left(R^{\prime}\left(G_{s}\right)\right)$ which form complete fundamental domain for the action of $G$ in $S^{3}=\partial \mathbf{H}^{4}$. From these formulas and the fact that the family $S$ constructed in Lemma 4 generates $G$ it follows that faces of $R(G)$ are matched by generators of $G$ and that the same is true for $R^{\prime}(G)$. The corollary is proved. Q.E.D.

Let $\langle\langle g\rangle\rangle_{G}$ be the normal closure of the infinite cycle group $\langle g\rangle$ in $G$.

Corollary 4.2. The group $G$ is isomorphic to the group $X /\left(\left\langle\left\langle g^{n}\right\rangle\right\rangle_{X}\right)$, where $X=$ $G_{s}^{\prime} * \mathscr{H}_{s}\left(\tau_{\sigma} G_{s}^{\prime} \tau_{\sigma}\right)$.

Proof. Each relation in the group $G$ now follows from an edge relation in the polyhedron $\mathscr{R} \subset \mathbf{H}^{4}$. Edges in $\partial \mathscr{R} \backslash\left\{\tilde{l}_{1}, \tilde{l}_{2}\right\}$ give us a system of generating relations of the group $X$. Additional relations come from edges $\tilde{l}_{i}$. These are just relations of the form $g^{n}=1$ and its consequences. The corollary is proved. Q.E.D.

Below we will use notations from Claim 2 of Lemma 3.

Lemma 5. The group $G$ contains a subgroup $G_{0}$ of finite index such that there is a normal nonelementary finitely generated subgroup $N_{0} \triangleleft G_{0}$ with infinite factor group $G_{0} / N_{0}$.

Proof. As we have shown in Lemma 3, the group $G_{s}$ is isomorphic to a subgroup $\widetilde{G}_{s} \subset R \subset \operatorname{Isom}\left(\mathbf{H}^{3}\right)$. By the same method we now prove that there is an isomorphism $i: X \rightarrow \tilde{X} \subset R \subset \operatorname{Isom}\left(\mathbf{H}^{3}\right)$ (for the definition of $X$ see Corollary 4.2 above). Indeed, there is a subgroup $\widetilde{\mathscr{H}}_{s} \subset R$ stabilizing a plane $\xi_{s} \subset B_{1}$ and hence $\widetilde{X}=i_{s}\left(\widetilde{G}_{s}^{\prime}\right) * \widetilde{\mathscr{H}}_{s} \tau_{\xi_{s}}\left(i_{s}\left(\widetilde{G}_{s}^{\prime}\right)\right) \tau_{\xi_{s}}$ is the required group.

We can easily obtain that $\Gamma_{0}$ and $\widetilde{X}$ are commensurable (see Lemma 3 ) and therefore there is a finitely generated subgroup $\tilde{Y}=\tilde{X} \cap F_{0}$ which is normal in $\widetilde{Q}=\widetilde{X} \cap \Gamma_{0}$ and $\widetilde{Q}$ is a subgroup of finite index in $\widetilde{X}, \widetilde{Q} / \widetilde{Y} \cong \mathbf{Z}$.

We recall that the isomorphism $i: X \rightarrow \widetilde{X}$ was constructed in such a way that $i \mid \Gamma_{1}^{\prime}=\mathrm{id}$. Thus, $i\left(g_{1}\right)=g_{1} \subset\left[F_{0}, F_{0}\right] \cap H_{1}^{\prime}$ (see Main Lemma). Hence, $g_{1} \in \widetilde{X} \cap\left[F_{0}, F_{0}\right] \subset \widetilde{Y}$. Let $\tilde{g}_{2}=\tau_{\xi_{s}} g_{1} \tau_{\xi_{s}}$. By using the normality of $\Gamma_{0}$ in $R$ one has $\tilde{g}_{2} \in F_{0}$ by the evident embedding $\tau\left(\left[F_{0}, F_{0}\right]\right) \tau^{-1} \subset F_{0}$ for any $\tau \in R$.

We can now say that $\left\langle\left\langle g_{1}, \tilde{g}_{2}\right\rangle\right\rangle_{\tilde{X}} \subset \widetilde{Y}$ because each element of the form $\tilde{x} g_{1} \tilde{x}^{-1}$ or $\tilde{x} \tilde{g}_{2} \tilde{x}^{-1}(\tilde{x} \in \tilde{X})$ belongs to $\tilde{X} \cap\left[F_{0}, F_{0}\right]$. Let $Y=i^{-1}(\tilde{Y})$, $Q=i^{-1}(\tilde{Q}), g_{2}=i^{-1}\left(\tilde{g}_{2}\right), g=g_{1}^{-1} g_{2}$.

By the considerations above $\left\langle\left\langle g^{n}\right\rangle\right\rangle_{X} \subset\left\langle\left\langle g_{1}, g_{2}\right\rangle\right\rangle_{X} \subset Y$. Let $\psi: X \rightarrow G$ be a natural projection and $\operatorname{Ker} \psi=\left\langle\left\langle g^{n}\right\rangle\right\rangle_{X} \subset Y$. The following diagram is then 
commutative

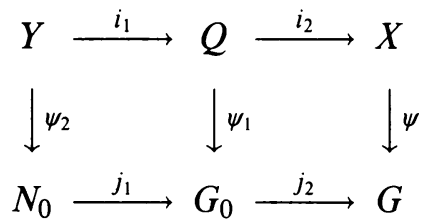

Here $i_{k}, j_{k}$ are inclusion maps $(k=1,2)$ and $\psi_{1}=\left.\psi\right|_{Q}, \psi_{2}=\left.\psi\right|_{Y}$ are restrictions of $\psi$.

Both groups $Q$ and $Y$ are finitely generated, so $N_{0}$ and $G_{0}$ are also finitely generated. Thus we have that $\operatorname{Ker} \psi=\operatorname{Ker} \psi_{2} \subset Y$ and obviously $Q / Y \cong$ $G_{0} / N_{0} \cong \mathbf{Z}$. Lemma 5 is proved. Q.E.D.

Lemma 6. The group $G$ contains no parabolics.

Proof. Each edge of $R(G)$ has a neighbourhood filled by a finite number of images of $R(G)$ under $G$ (see the proof of Lemma 4). Thus, the stabilizer of each point of $\partial R(G)$ is at most finite.

Recall that the circle $l_{1}=I_{g_{1}} \cap I_{g_{2}}$ is pointwise fixed under the elliptic element $g=g_{1}^{-1} g_{2}$ of order $n$. There exists a small neighbourhood $V_{1}=N\left(l_{1}\right)$ of $l_{1}$, invariant under $g$ for which $V_{1} \cap I_{\gamma}=\varnothing, \gamma \in\left(\Gamma_{1}^{\prime} \cup \tau_{\sigma} \Gamma_{1}^{\prime} \tau_{\sigma}\right) \backslash\left\{g_{1}, g_{2}\right\}$.

Therefore, by construction, $R(G) \cap V_{1}=\mathscr{P}(\langle g\rangle) \cap V_{1}$ where $\mathscr{P}(\langle g\rangle)$ is an isometric fundamental domain for the cyclic group $\langle g\rangle$. It now follows that $\gamma V_{1} \cap V_{1}=\varnothing, \gamma \in G \backslash\langle g\rangle$. Let us consider the domain $\Omega_{G}^{-}=\Omega_{G} \backslash G V_{1}$ and surface $\sigma^{\prime}=\operatorname{cl}\left(\sigma \cap \Omega_{G}^{-}\right)$. It is not hard to see that $\sigma^{\prime}$ is strongly invariant under $\mathscr{H}_{s}$ in $G, \mathscr{H}_{s}=\operatorname{Stab}\left(\Pi_{s}, G_{s}\right)$.

One can write each $f \in G \backslash \mathscr{H}_{s}$ in a normal form, $f=f_{1} \cdot f_{2} \cdots \cdots f_{m}$, where $f_{i}$ belongs either to $G_{s}^{\prime} \backslash \mathscr{H}_{s}$ or to $\left(\tau_{\sigma} G_{s}^{\prime} \tau_{\sigma}\right) \backslash \mathscr{H}_{s}, G=\left\langle G_{s}^{\prime}, \tau_{\sigma} G_{s}^{\prime} \tau_{\sigma}\right\rangle$. Suppose that $f$ is of infinite order; we get $f\left(\operatorname{int}\left(\sigma^{\prime}\right)\right) \subset$ int $\sigma^{\prime}$ if $m$ is even. Evidently $\sigma^{\prime}$ divides $\Omega_{G}^{-}$; let int $\sigma^{\prime}$ (respectively ext $\sigma^{\prime}$ ) be the bounded (resp. unbounded) component of $\Omega_{G}^{-} \backslash \sigma^{\prime}$. We have $f^{2 n}\left(\right.$ int $\left.\sigma^{\prime}\right) \subset f^{2 n-2}\left(\right.$ int $\left.\sigma^{\prime}\right)$ and $f^{-2 n}\left(\operatorname{ext} \sigma^{\prime}\right) \subset$ $f^{-2 n+2}\left(\operatorname{ext} \sigma^{\prime}\right)$. So $x=\lim _{n \rightarrow \infty}\left(f^{2 n}\left(\sigma^{\prime}\right)\right)$ and $y=\lim _{n \rightarrow \infty} f^{-2 n}\left(\sigma^{\prime}\right)$ are respectively the attractive and the repulsive fixed points of $f(n \in \mathbf{N})$.

Let us prove that $x \neq y$. Indeed, as we have seen, $\operatorname{cl} V_{1} \subset \Omega(G)$ so $\{x, y\} \cap G\left(V_{1}\right)=\varnothing$. We conclude that the points $x, y$ are separated by $\sigma$ and $\operatorname{dist}(x, y)>0$. The lemma is proved. Q.E.D.

Lemma 7. The domain of discontinuity $\Omega(G)$ of the group $G$ consists of two invariant components $\Omega_{G}$ and $\Omega_{G}^{\prime}\left(\infty \in \Omega_{G}\right)$.

Proof. We have shown in Corollary 1 to Lemma 4 that the fundamental domain of the group $G$ consists of two connected components $R(G)$ and $R^{\prime}(G)$. Furthermore, generators of $G$ independently match faces of $R(G)$ with faces of $R(G)$ and faces of $R^{\prime}(G)$ with faces of $R^{\prime}(G)$.

Write $\Omega_{G}=G \cdot R(G), \Omega_{G}^{\prime}=G \cdot R^{\prime}(G)$ for the unions of $G$-translates of $R(G)$ and $R^{\prime}(G)$. We obtain $\Omega_{G} / G=R(G) / \sim=O$ and $\Omega_{G}^{\prime} / G=R^{\prime}(G) / \sim=O^{\prime}$, where the second quotient in each expression means an orbifold obtaining by identifying paired faces of the fundamental polyhedron.

The group $G$ is geometrically finite, since its 4-dimensional fundamental polyhedron $\mathscr{R} \subset \mathbf{H}^{4} \cup \Omega(G)$ has finitely many faces by the formula (*) (see the proof of Lemma 4). By Lemma 6 the group $G$ contains no parabolics, so we can conclude that both $O$ and $O^{\prime}$ are compact orbifolds. 


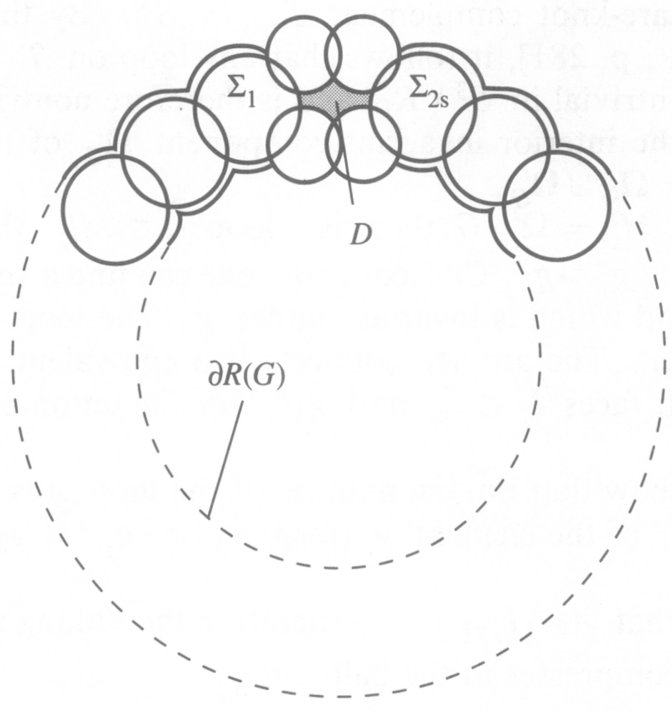

FIGURE 4

Since $R(G)$ and $R^{\prime}(G)$ together form a fundamental domain for $G$ in $S^{3}$, the domains $\Omega_{G}$ and $\Omega_{G}^{\prime}$ are disjoint and $\Omega(G)=\Omega_{G} \cup \Omega_{G}^{\prime}$. Notice that the domain $\Omega_{G}$ (respectively $\Omega_{G}^{\prime}$ ) is connected because faces of $R(G)$ (resp. $R(G)$ ) are equivalent under $G$ to faces of $R(G)$ (resp. $R^{\prime}(G)$ ).

Now standard arguments show that any boundary point of one of the components $\Omega_{G}$ and $\Omega_{G}^{\prime}$ is a limit point. Indeed, let $x$ be a boundary point of (say) $\Omega_{G}$ then there is a sequence of different points $\left(x_{n}\right)$ in $\Omega_{G}$ tending to $x$. By compactness of $O$ and the fact that $\Omega_{G} \rightarrow R(G) / \sim$ is a covering projection [M2, p. 68] we observe that all but finitely many $x_{n}$ are equivalent under $G$. Consequently, $x \in \Lambda(G)$. The lemma is proved. Q.E.D.

Proof of Theorem B. In Lemma 5, we have obtained an element $g \in N_{0}$ of finite order (in fact $g \in\left[N_{0}, N_{0}\right]$ ).

Let us suppose that $z \in G_{0} \backslash N_{0}$ projects to a generator of the group $G_{0} / N_{0}$. In $N_{0}$ there are infinitely many nonconjugate elements of the form $\gamma_{m}=$ $z^{m} g z^{-m} \quad(m \geq 0)$. Indeed, each $\gamma_{m}$ fixes pointwise a single circle $l_{m} \subset \Omega_{G_{0}} \subset$ $S^{3}$. If $\gamma_{m}$ was conjugate by $a \in N_{0}$ to $\gamma_{n}$, then the element $a$ would move $l_{m}$ to $l_{n}$ and the same would be true for the element $z^{-m+n}$. This would mean that $\left(z^{-m+n}\right) a^{-1}$ has finite order and so $z^{p} \in N_{0}$ for some $p \geq 0$ (since $N_{0}$ is normal in $G_{0}$ ), which is impossible. Theorem B is proved.

The last step in the proof of Theorem A is the following.

Lemma 8. The component $\Omega_{G}$ of the domain of discontinuity $\Omega(G) \quad\left(\infty \in \Omega_{G}\right)$ contains an incompressible torus $T \subset \operatorname{int} R(G) \subset \Omega_{G}$.

Proof. The boundary $\partial R(G)$ is a connected sum of two tori and so is a surface of genus 2 (see Figure 4). There is a compression disk $D \subset R(G)$ shown on the Figure 4. Now we denote by $V(D)$ the regular neighbourhood of $D$ and let $R_{D}=\operatorname{cl}(R(G) \backslash V(D))$.

The proof of the lemma consists in showing that the boundary of the torus $T=\partial R_{D}$ is incompressible in $\Omega_{G}$. 
We have a square-knot complement $R_{D}$ in $S^{3}$. By the property $P$ for nonprime knots [R, p. 281], it follows that any loop on $T$ different from the meridian $m$ is nontrivial in $S^{3} \backslash R_{D}$ and is therefore nontrivial in $\Omega_{G} \backslash R_{D}$.

Now consider the interior invariant component $\Omega_{G}^{\prime}$ of the domain of discontinuity $\Omega(G)=\Omega_{G} \cup \Omega_{G}^{\prime}$.

In the quotient $M_{G}^{\prime}=\Omega_{G}^{\prime} / G$ there is a loop $\alpha \subset M_{G}^{\prime}$ which represents the elliptic element $g=g_{1}^{-1} \cdot g_{2}$. Consequently, one can find a simple loop $A \subset \Omega_{G}^{\prime}$ which covers $\alpha$ and which is invariant under $g$. The loop $A$ contains an arc $A_{1}$ projecting to $\alpha$. The arc $A_{1}$ connects two equivalent points $y_{1}$ and $y_{2}$ lying on equivalent faces $\xi_{2} \subset I_{g_{2}}$ and $g\left(\xi_{2}\right)$ of the union of two polyhedrons $R^{\prime}(G) \cup g\left(R^{\prime}(G)\right)$.

Below we will show that linking number of the loop $A$ with the fixed circle $l_{1}$ (respectively $l_{2}$ ) of the element $g$ (resp. $g_{1} \cdot g \cdot g_{1}^{-1}=g_{2} \cdot g_{1}^{-1}$ ) is equal to 1 (resp. 0).

We can ensure that $A_{1} \cap I_{g_{1}^{-1}}=\varnothing$. Therefore the linking number of $A$ with $l_{2}$ is 0 , since $l_{2}$ compresses in the ball $\operatorname{int}\left(I_{g_{1}^{-1}}\right)$.

Let us now show that the linking number of $A$ with $l_{1}$ is equal to 1 . Consider the covering projection $S^{3} \backslash l_{1} \stackrel{\pi}{\rightarrow}\left(S^{3} \backslash l_{1}\right) /\langle g\rangle=M_{g}$. The group $\pi_{1}\left(M_{g}\right)$ is an extension of $\mathbf{Z}_{n}$ by $\mathbf{Z}$ and is torsion-free. As the arc $A_{1}$ projects to nontrivial loop in $M_{g}$, the loop $\pi(A)$ is also nontrivial; otherwise, by connectedness of $A$, we would have an element of finite order in $\pi_{1}\left(M_{g}\right)$. It now follows that $A$ is nontrivial in $S^{3} \backslash l_{1}$ and thus that the linking number $A$ with $l_{1}$ is equal to 1 ( $A$ is simple loop).

Therefore, the meridian $m$ (which is freely homotopic to $l_{1} \cdot l_{2}$ ) has linking number 1 with $A$. Observe that the meridian $m \subset \Omega_{G}$ and the loop $A \subset \Omega_{G}^{\prime}$ are separated by the limit set $\Lambda(G)$ so they cannot be trivial in both of these components. We have shown that $m$ is nontrivial in $\Omega_{G} \backslash R_{D}$; the lemma is proved. Q.E.D.

Proof of Theorem A. By the Selberg Lemma [Se], there exists a torsion-free subgroup $G^{*} \subset G$ of finite index; let $N=G^{*} \cap N_{0}$. We have an exact sequence of finitely generated groups

$$
1 \rightarrow N \rightarrow G^{*} \rightarrow \mathbf{Z} \rightarrow 1 .
$$

and a diagram of infinite regular coverings

$$
\Omega_{G} \rightarrow \Omega_{G} / N \rightarrow \Omega_{G} / G^{*}
$$

since $\Omega(G)=\Omega\left(G^{*}\right)=\Omega(N)$. The manifold $M_{G^{*}}=\Omega_{G} / G^{*}$ is compact so, if the group $\pi_{1}\left(M_{N}=\Omega_{G} / N\right)$ is finitely generated, then (by [H, Theorem 11.1]) $\pi_{1} M_{N}$ is isomorphic to the fundamental group of a surface.

By Lemma $8, \pi_{1} M_{N}$ contains a subgroup isomorphic to $\mathbf{Z} \oplus \mathbf{Z}$, so it should be a finite extension of the latter. This is impossible, since $N$ is not an elementary group. Theorem A is proved. Q.E.D.

\section{APPENDIX}

Below we will present some complementary results. Recall that the group $N_{0}$ constructed in Theorem B contains infinitely many nonconjugate elliptic elements $\gamma_{m}$, all having the same order $n$. By our construction (see the beginning of $\S 4$ ) we can assume that $n$ is prime. 
Assertion 1. ${ }^{3}$ The group $N$ is not finitely presented.

Proof. Suppose to the contrary that the torsion-free subgroup $N \subset N_{0}$ is finitely presented. Then $N_{0}$ would also be finitely presented; here $N_{0} / N \cong K$ is a finite group. The group $K$ acts on the manifold $M(N)=\mathbf{H}^{4} / N$, so that $M\left(N_{0}\right)=$ $M(N) / K$. Consider the set $L$ of fixed circles $\tilde{l}_{n} \subset \mathbf{H}^{4}$ of the elements $\gamma_{m}$. No elements of $\left\{\gamma_{m}\right\}$ are conjugate in $N_{0}$, so $L$ projects to a set $L^{\prime} \subset M(N)$ containing infinitely many different components, each fixed by an element of $K$ whose order is $n$. Since $K$ is finite, there is $k \in K$ of prime order which fixes infinitely many different circles in $L^{\prime}$.

We now claim that the group $H_{3}(N, \mathbf{Z})$ is infinitely generated. Otherwise, all groups $H_{i}(M(N), \mathbf{Z}) \quad(i=1, \ldots, 4)$ would be finitely generated, since the manifold $M(N)$ is not compact and $N$ is finitely presented. By Smith theory, it follows that the cohomology groups of the fixed-point set of the element $k$ of prime order $n$ acting on $M(N)$ would all be finitely generated (see e.g. [Br, Theorem VII.10.5, p. 181-182]), so $k$ could not fix infinitely many circles in $L^{\prime}$. Therefore, $H_{3}(N, \mathbf{Z})$ is infinitely generated. Notice that the group $H_{3}(M(N), Z)$ cannot contain infinitely many linearly independent elements represented by nonseparated submanifolds of $M(N)$, otherwise, by duality, the group $H_{1}(M(N), \mathbf{Z})$ would also be infinitely generated. We would then conclude that the manifold $M(N)$ has infinitely many ends; let us show that this cannot happen.

The group $N$ is a normal subgroup in the geometrically finite subgroup $G$ having two invariant components of its action on $S^{3}$ (Lemma 7). There are two corresponding components (say $L_{1}$ and $L_{2}$ ) of the boundary of the convex hull $H_{G} \subset \mathbf{H}^{4}$ of the limit set $\Lambda(G)$. Between $L_{1}$ and $\Omega_{G}$ is an open region $E_{1}$; similarly we obtain $E_{2}$. The fundamental group of either component of the boundary of the convex hull maps onto $G$. It follows that there is a constant $\mathscr{C}$ such that, in the universal cover, every point of the convex hull is within distance $\mathscr{C}$ of each of these components of the boundary of the convex hull. Let us remove a compact set $K$ from $M(N)$, then the unbounded components of $E_{i} / N \backslash K \quad(i=1,2)$ lie in the same component of the complement of $K$, because they can be joined by an arc of length $\mathscr{C}$ taken arbitrarily far from $K$. If there was another unbounded component of the complement then it would be separated from the component $M(N) \backslash K$ containing $E_{i} / N \backslash K$ by a compact surface $S$. We get a contradiction: points arbitrarily far away from $S$ and, thus from the boundary of the convex hull lie in neither the set $E_{1} / N$ nor $E_{2} / N$. The assertion is proved. Q.E.D.

In the following assertion we assume that $M=\mathbf{H}^{3} / \Gamma$ is a closed hyperbolic 3-manifold which fibers over the circle with a fiber - closed surface $S_{g}(g>1)$.

Assertion 2. Suppose that the group $\Gamma$ above is commensurable with a reflection group $R$ in faces of a polyhedron $D \subset \mathbf{H}^{3}$ all of whose dihedral angles are right angles. Then there is a finite-index subgroup $\Gamma_{0} \subset \Gamma$ and a 1-parameter family of discrete faithful representations $\rho(t): \Gamma \rightarrow M(3) \cong S O_{+}(1,4) \quad(t \in[-\varepsilon, 0])$ converging to a representation $\rho_{0}$ so that

1. All groups $G(t)=\rho_{t}\left(\Gamma_{0}\right)$ are convex cocompact.

2. The group $G(0)=\rho_{0}\left(\Gamma_{0}\right)$ is Kleinian with domain of discontinuity con-

\footnotetext{
${ }^{3}$ The author wishes to thank the referee for pointing out to him the proof of Assertion 1.
} 
sisting of two invariant components (noncontractible) $\Omega_{G(0)}$ and $\Omega_{G(0)}^{*} \quad(\infty \in$ $\Omega_{G(0)}$.

3. The normal subgroup $F(0)=\rho\left(\Gamma_{0} \cap \pi_{1} S_{g}\right)$ of $G(0)$ acts freely on $\Omega_{G(0)}$ so that the fundamental group $\pi_{1}\left(\Omega_{G(0)}\right) / F(0)$ is infinitely generated. Moreover, $F(0)$ is isomorphic to the fundamental group of a closed surface but contains infinitely many $F(0)$-conjugacy classes of maximal parabolic subgroups.

Proof. We start with the group $G_{s}$ and its fundamental domain $R\left(G_{s}\right)$ from Lemma 3. Now take a family of spheres $\sigma_{t}(t \in[-\varepsilon, 0])$ such that $\sigma_{t} \cap \Sigma_{s}=\Lambda_{s}$, $\sigma_{t} \subset \pi^{-}$for $t \leq 0$; and $\sigma_{t} \cap I_{g_{i}}=\varnothing \quad(t<0)$, with $\sigma_{0}$ touching $I_{g_{i}}$ in a point $p_{i}(i=1,2)$.

If now $t<0$, we put $\Omega_{s}^{-}=\Omega_{G_{s}} \backslash G \cdot \operatorname{int}\left(\sigma_{t}\right)$ and $G_{s}^{\prime}=\operatorname{Stab}\left(\Omega_{s}^{-}, G_{s}\right)$. The required group $G(t)=\left\langle G_{s}^{\prime}, \tau_{\sigma_{t}} G_{s}^{\prime} \tau_{\sigma_{t}}\right\rangle$ is discontinuous and convex cocompact (by the proof of Lemma 3).

If $t=0$, the construction of $G(0)$ is analogous to that of $G$ from Lemma 4 (here we use Poincaré's Polyhedron Theorem to obtain $G(0)$ ). But the group $G(0)$ contains a parabolic element $g(0)=g^{-1} \cdot g_{2}(0)$, where $g_{2}(0)=\tau_{\sigma_{0}} \cdot g_{1} \cdot \tau_{\sigma_{0}}$ fixes the point $p$. The assertion is proved. Q.E.D.

We notice that in [P1] we constructed limits of 2-quasifuchsian groups with properties 1-2 from assertion 2 with $\Gamma$ satisfying somewhat weaker conditions.

\section{REFERENCES}

[A-M] W. Abikoff and B. Maskit, Geometric decomposition of Kleinian groups, Amer. J. Math. 99 (1977), 687-697

[A] L. V. Ahlfors, Finitely generated Kleinian groups, Amer. J. Math. 86 (1964), 413-429; 87 (1965), 759.

[Be-M] A. F. Beardon and B. Maskit, Limit points of Kleinian groups and finite-sided fundamental polyhedra, Acta Math. 132 (1974), 1-12.

[B] F. Bonahon, Bouts des varietes hyperboliques de dimension 3, Ann. of Math. 124 (1986), 71-158.

[Bow-M] B. Bowditch and G. Mess, A 4-dimensional Kleinian group, preprint

[F-M] M. Feighn and G. Mess, Conjugacy classes of finite subgroups of Kleinian groups, Amer. J. Math. (to appear).

[G] M. Gromov, Hyperbolic groups, Essays in Group Theory (S.M. Gerstein, ed.), M.S.R.I. Publ. N8, 1988.

[H] J. Hempel, 3-manifolds, Princeton Univ. Press, Princeton, N.J., 1976.

[J] T. Jørgensen, Compact 3-manifolds of constant negative curvature fibering over the circle, Ann. of Math. (2) 106 (1977), 61-72.

[K] M. Kapovich, On absence of Sullivan's cusp finiteness theorem in higher dimensions, IHES, preprint, 1991.

[K-P] M. Kapovich and L. Potyagailo, On absence of Ahlfors finiteness theorem for Kleinian groups in dimension 3, Topology Appl. 40 (1991), 83-91.

[Kra] I. Kra, Automorphic forms and Kleinian groups, Benjamin, Reading, Mass., 1972.

[Ku-S] R. Kulkarni and P. Shalen, On Ahlfors' finiteness theorem, Adv. Math. 76 (1989), 155-169.

[L] D. Long, Immersions and embeddings of totally geodesic surfaces, Bull. London Math. Soc. 19 (1987), 481-484.

[Mal] A. I. Maltsev, On the faithful representations infinite groups by matrices, Mat. Sb. 8(50) (1940), 405-422; English transl., Amer. Math. Soc. Transl. (2) 45 (1965), 1-18.

[M1] B. Maskit, On Klein combination theorem III, Advances in the Theory of Riemann Surfaces, Princeton Univ. Press, Princeton, N.J., 1971, pp. 297-310. 
[M2] _ Kleinian groups, Springer-Verlag, 1988.

[Mats] S. Matsumoto, Foundations of flat conformal structures, preprint.

[Mc] D. McCullough, Compact submanifolds of 3-manifolds with boundary, Quart. J. Math. (2) 37 (1986), 299-307.

[Mc-F] D. McCullough and M. Feighn, Finiteness conditions for 3-manifolds with boundary, Amer. J. Math. 109 (1987), 1155-1169.

[P] L. Potyagailo, The problem of finiteness for Kleinian groups in 3-space, Proc. Internat. Conf. “Knots-90", (Osaka, 1990), edited by A. Kawauchi, De Gruyter, 1992, pp. 619-623.

[R] D. Rolfsen, Knots and links, Publish or Perish, 1976.

[Sc1] P. Scott, Finitely generated 3-manifold groups are finitely presented, J. London Math. Soc. (2) 6 (1973), 437-440.

[Sc2] _ Subgroups of surface groups are almost geometric, J. London Math. Soc. 17 (1978), 555-565; Correction, ibid 32 (1985), 217-220.

[Se] A. Selberg, On discontinuous groups in higher dimensional symmetric spaces, Contribution to Function Theory, Bombay, 1960, pp. 147-164.

[Su] D. Sullivan, Travaux de Thurston sur les groupes quasi-fuchsiens et les varietes hyperboliques de dimension 3 fibres sur $S^{1}$, Lecture Notes in Math., vol. 842, Springer, Berlin and New York, 1981, pp. 196-214.

[Su2] _ A finiteness theorem of cusps, Acta Math. 147 (1981) 289-299.

[T1] W. Thurston, The geometry and topology of 3-manifolds, Princeton Univ. Lecture Notes, Princeton, N.J., 1978.

[T2] - Hyperbolic structures on 3-manifolds I: Deformation of acylindrical manifolds, Ann. of Math. 124 (1986), 203-246; II: Surface groups and 3-manifolds which fibers over the circle, preprint.

[Tu] P. Tukia, On isomorphisms of geometrically finite Möbius groups, Publ. Math. Inst. Hautes Etudes Sci. 61 (1985), 171-214.

U.F.R. de Mathématiques, Université de Lille 1, 59655 Villeneuve d’AscQ Cedex, FRANCE

E-mail address: potyag@gat.univ-lille1.fr 